\title{
Cosine Modulated Multitone for Very High-speed Digital Subscriber Lines
}

\author{
Lekun Lin and Behrouz Farhang-Boroujeny \\ The Department of Electrical and Computer Engineering \\ University of Utah \\ e-mails: llin@eng.utah.edu and farhang@ece.utah.edu
}

\begin{abstract}
In this paper, the use of cosine modulated filter banks (CMFBs) for multi-carrier modulation in the application of very high-speed digital subscriber lines (VDSL) is studied. We refer to this modulation technique as cosine modulated multitone (CMT). CMT is fundamentally the same as the discrete wavelet multitone (DWMT) which has been known since 1993 and is well studied in the literature. In fact, both methods transmit the same modulated signals. However, the receiver structure in CMT is different from its DWMT counterpart. DWMT uses linear combiner equalizers that combine signals from each sub-carrier band and its adjacent bands. Such equalizers are relatively complex - typically more than 20 taps per sub-carrier are required. CMT, on the other hand, adopts a receiver structure that uses only two taps per sub-carrier for equalization.

This paper has the following contributions. (i) The use of CMFB for multi-carrier communication with a simplified equalizer has recently been proposed. A modification to the structure of the receiver that further reduces its complexity is proposed. (ii) Although traditionally CMFBs are designed to satisfy perfect reconstruction $(\mathrm{PR})$ property, in transmultiplexing applications, the presence of channel destroys the PR property of the filter bank, and thus other criteria of filter design should be adopted. We propose one such method. (iii) Through extensive computer simulations, we compare CMT with zipper discrete multitone (z-DMT) and filtered multitone (FMT), the two modulation techniques that have been included in the VDSL draft standard. Comparisons are made in terms of computational complexity, transmission latency, achievable bit-rate and resistance to radio ingress noise. In terms of computational complexity, z-DMT is found superior to CMT and FMT. CMT, on the other hand, offers the highest bit rate. Both CMT and FMT outperform z-DMT with respect to transmission latency and resistance to radio ingress noise. CMT is significantly less complex than FMT.
\end{abstract}

Keywords: VDSL, Cosine modulated filter banks, Cosine modulated multitone, Wavelet multitone 


\section{INTRODUCTION}

In recent years, multi-carrier modulation (MCM) has attracted considerable attention as a practical and viable technology for high-speed data transmission over spectrally shaped noisy channels [1]-[6]. The most popular MCM technique uses the properties of the discrete Fourier transform (DFT) in an elegant way so as to achieve a computationally efficient realization. Cyclic prefix (CP) samples are added to each block of data to resolve and compensate for channel distortion. This modulation technique has been accepted by standardization bodies in both wired (digital subscriber lines - DSL) [7]-[10] and wireless [11], [12] channels. While the terminology discrete multitone (DMT) is used in the DSL literature to refer to this MCM technique, in wireless applications the terminology orthogonal frequency division multiplexing (OFDM) has been adopted. The difference is that in DSL applications MCM signals are transmitted at baseband, while in wireless applications MCM signals are up-converted to a radio frequency (RF) band for transmission.

Zipper DMT (z-DMT) is the latest version of DMT that has been proposed as an effective frequency division duplexing (FDD) method for very high-speed DSL (VDSL) applications. Two variations of z-DMT have been proposed: (i) synchronous zipper [13], [14] and (ii) asynchronous zipper [15]. The synchronous zipper requires synchronization of all modems sharing the same cable (a bundle of twisted pairs). As this is found too restrictive (many modems have to be synchronized), it has been identified as an infeasible solution. The asynchronous zipper, on the other hand, at the cost of some loss in performance, requires only synchronization of the pairs of modems that communicate with each other. The unsynchronized modems on the same cable then introduce some undesirable cross-talk noise. Since the asynchronous z-DMT is the one that has been adopted in the VDSL draft standard [37], in the rest of this paper all references to z-DMT are with respect to its asynchronous version.

To synchronize a pair of modems in z-DMT, cyclic suffix (CS) samples are used. Moreover, to suppress the side-lobes of DFT filters and thus allow more effective FDD, extensions are made to the CP and CS samples and pulse shaping filters are applied [15]. All these add to the system overhead and thus reduce the bandwidth efficiency of z-DMT.

Radio frequency interference (RFI) is a major challenge that any VDSL modem has to deal with. RF signals generated by amateur radios (HAM signals) coincide with the VDSL band [3], [4]. Thus, there is a potential of interference between VDSL and HAM signals. The first solution to separate HAM and VDSL signals is to prohibit VDSL transmission over the HAM bands. This solution along with the pulse shaping method adopted in z-DMT will solve the problem of VDSL signals egress interference with HAM signals. However, the poor side-lobe behavior of DFT filters and also the very high level of RFI still result in interference which degrades the performance of z-DMT significantly. RFI cancellers are thus needed to improve the performance of z-DMT. There are a number of methods in the literature that cancel RFI by treating the ingress as a tone with no or very small variation in amplitude over each data block of DMT [16], [17], [18]. Such methods have been found to be limited in performance. Another method is to pick up a reference RFI signal from the common mode component of the twisted-pair signals and use it as input to an adaptive filter for synthesizing and subtracting the RFI from the received signal [19]. This method which may be implemented in analog or digital form can suppress RFI by as much as 20 to $25 \mathrm{~dB}$ [18]. Our understanding from the limited literature available on RFI cancellation is that a combination of these two methods will result in the best performance in any DMT-based transceiver. Thus, the comparisons given in the later sections of this paper consider such a RFI canceller setup for z-DMT.

Since RFI cancellation is rather difficult to implement, there is a current trend in the industry to adopt filter bank-based MCM techniques. These can deal with RFI more efficiently, thanks to much superior stop-band suppression behavior of filter banks compared to DFT filters. We note that z-DMT has made an attempt to improve on stop-band suppression. However, as we show in Section VI, z-DMT is still much inferior to filter bank solutions. 
Filtered multitone (FMT) is a filter bank solution that has been proposed by IBM [34], [35], [36] and has been widely studied recently. In order to avoid interference among various sub-carriers, FMT adopts a filter bank with very sharp transition bands and allocates sufficient excess bandwidth, typically in the range from 0.05 to 0.125 . This introduces significant inter-symbol interference (ISI) that is dealt with by using a separate decision feedback equalizer (DFE) for each sub-carrier [36]. Such DFEs are computationally very costly as they require relatively large number of feed-forward and feedback taps. Nevertheless, the advantages offered by this solution, especially with respect to suppression of ingress RFI, has justified its application and thus FMT has been included as an annex to the VDSL draft standard [37].

Cosine modulated filter banks (CMFB) working at maximally decimated rate, on the other hand, are well understood and widely used for signal compression [28]. Moreover, the use of filter banks for realization of transmultiplexer systems [28] as well as their application to MCM [22] have been recognized by many researchers. In particular, the use of CMFB to multi-carrier data transmission in DSL channels has been widely addressed in the literature, under the common terminology of discrete wavelet multitone (DWMT), e.g., [20]-[27]. In DWMT, it is proposed that channel equalization in each sub-carrier be performed by combining the signals from the desired band and its adjacent bands. These equalizers that have been referred to as post-combiner equalizers impose significant load to the computational complexity of the receiver. This complexity and the lack of an in-depth theoretical understanding of DWMT have kept industry lukewarm about it in the past.

A revisit to CMFB-MCM/DWMT has been made recently [39], [40], [41], [42]. In the first work, [39], an in-depth study of DWMT has been performed, assuming that the channel could be approximated by a complex constant gain over each sub-carrier band. This study, which is also intuitively sound, revealed that the coefficients of each post-combiner equalizer are closely related to the underlying prototype filter of the filter bank. Furthermore, there are only two parameters per sub-carrier that need to be adapted; namely, the real and imaginary parts of the inverse of channel gain. In a further study [40], [41], it was noted that by properly restructuring the receiver, each post-combiner equalizer could be replaced by a two tap filter. It was also shown that there is no need for cross-filters (as used in the post-combiner equalizers in DWMT), thanks to the (near) perfect reconstruction property of CMFB. Moreover, a constant modulo blind equalization algorithm (CMA) was developed [40], [41]. In [42] also a receiver structure that combines signals from a CMFB and a sine modulated filter bank (SMFB) is proposed to avoid cross filters. This structure which is fundamentally similar to the one in [40], [41] approaches the receiver design from a slightly different angle. The complexity of CMFB/SMFB receiver is discussed in [43] where an efficient structure is proposed. In a further development [44], it is noted that CMFB/SMFB can be configured for transmission of complex modulated (such as QAM - quadrature amplitude modulated) signals. This is useful for data transmission over RF channels, but is not relevant to xDSL channels which are fundamentally baseband.

In this paper, we extend the application of CMFB-MCM to VDSL channels. The following contributions are made. The receiver structure proposed in [40], [41] is modified in order to minimize its computational complexity. Moreover, we discuss the problem of prototype filter design in transmultiplexer systems. We note that the traditional perfect reconstruction (PR) designs are not appropriate in this application and thus develop a novel near PR (NPR) design strategy. We contrast the CMFB-MCM against z-DMT and FMT and make an attempt to highlight the relative advantages that each of these three methods offer. In order to distinguish between the proposed method and DWMT, we refer to it as cosine modulated multitone (CMT). We believe the term 'cosine modulated filter bank' (and thus CMT) is more reflective of the nature of this modulation technique than the term 'wavelet'. The term wavelet is commonly used in conjunction with filter banks in which the bandwidth of each sub-band varies proportional to its center frequency. In CMFB, all sub-bands have the same bandwidth. Moreover, the modulator and demodulator blocks 
that we use are directly developed from a pair of synthesis and analysis CMFB, respectively. We should also acknowledge that there have been some attempts to develop communication systems that use wavelets with variable bandwidths, e.g., see [38] and the references therein.

An important class of filter bank based transmultiplexer systems that avoid ISI and ICI completely have been studied recently, e.g., [45] and [46]. Similar to DMT, where cyclic prefix samples are used to avoid ISI and ICI, here also redundant samples are added (through precoding, for example) for the same purpose. Such systems, thus, similar to DMT and FMT, suffer from bandwidth loss/inefficiency. Moreover, since the designed filter banks, in general, are not based on a prototype filter, they cannot be realized in any simple manner, e.g., in a polyphase DFT structure. Hence, they do not seem attractive for applications such as DSL where filter banks with a large number of sub-bands have to be adopted.

The rest of this paper is organized as follows. We present an overview of CMFB-MCM/CMT in Section II. In Section III, we propose a novel structure of CMT receiver which reduces its complexity significantly compared to the previous reports [40], [41]. In Section IV, we develop a novel method of prototype filter design. Computational complexities and latency issues are discussed and comparisons with z-DMT and FMT are made in Section V. This will be followed by a presentation of a wide range of computer simulations, in Section VI, where we compare z-DMT, FMT and CMT under different practical conditions. The concluding remarks are made in Section VII.

\section{Cosine Modulated Multitone}

Fig. 1 presents block diagram of a CMFB-based transmultiplexer system. At the transmitter, the data symbol streams, $s_{k}(n)$, are first expanded to a higher rate by inserting $M-1$ zeros after each sample. Modulation and multiplexing of data streams are then done using a synthesis filter bank. At the receiver, an analysis filter bank followed by a set of decimators are used to demodulate and extract the transmitted symbols. The delay $\delta$ at the receiver input is required to adjust the total delay introduced by the system to an integral multiple of $M$. When $\delta$ is selected correctly, channel noise, $\nu(n)$, is zero and the channel is perfect, i.e., $H(z)=1$, a well designed transmultiplexer delivers a delayed replica of data symbols $s_{k}(n)$ at its outputs, i.e., $\hat{s}_{k}(n)=s_{k}(n-\Delta)$, where $\Delta$ is an integer. However, due to the channel distortion, the recovered symbols suffer from intersymbol interference (ISI) and inter-carrier interference (ICI). Equalizers are thus used to combat the channel distortion. As noted above, post-combiner equalizers that span across the adjacent sub-bands and along the time axis were originally proposed for this purpose [22]. Such equalizers are rather complex - typically, 20 or more taps per sub-carrier are used. A recent development [40], [41] has shown that with a modified analysis filter bank, each sub-carrier can be equalized by using only two taps. In the rest of this section, we present a review of this modified CMFB-based transmultiplexer and explain how such simple equalization can be established. As noted above, we call this new scheme CMT.

In CMT, the transmitter follows the conventional implementation of synthesis CMFB [28]. For the receiver, we resort to a non-simplified structure of the analysis CMFB. Fig. 2 presents a block diagram of this non-simplified structure for an $M$-band analysis CMFB; see [28] for development of this structure. $G_{k}(z), 0 \leq k \leq 2 M-1$, are the polyphase components of the filter bank prototype filter $P(z)$, viz.,

$$
P(z)=\sum_{k=0}^{2 M-1} z^{-k} G_{k}\left(z^{2 M}\right) .
$$

The coefficients $d_{0}, d_{1}, \cdots, d_{2 M-1}$ are chosen in order to equalize the group delay of the filter bank sub-channels. This gives $d_{k}=e^{j \theta_{k}} W_{2 M}^{(k+0.5) N / 2}$ for $k=0,1, \cdots, M-1$, and $d_{k}=d_{2 M-1-k}^{*}$ for $k=M, M+1, \cdots, 2 M-1$, where $\theta_{k}=(-1)^{k} \frac{\pi}{4}, W_{2 M}=e^{-j 2 \pi / 2 M}, *$ denotes conjugate, and $N$ is the order of $P(z)$. 
Transmitter

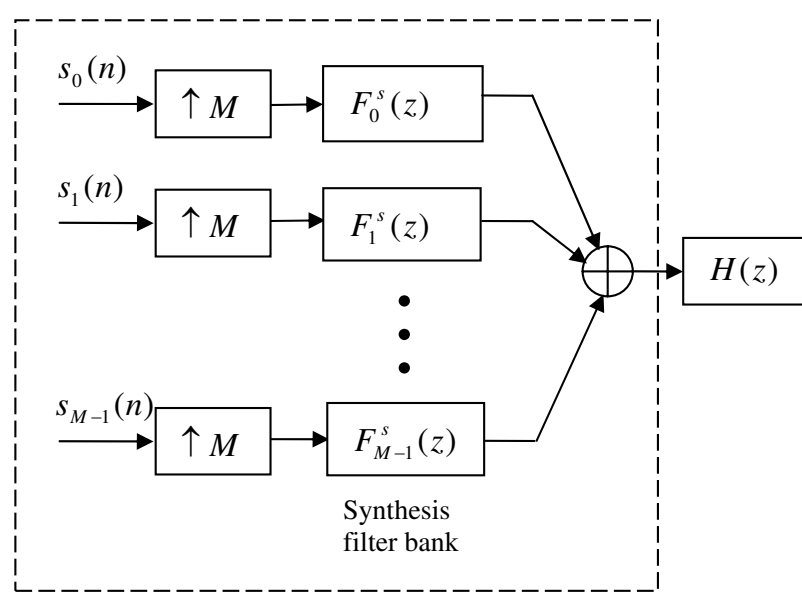

Receiver

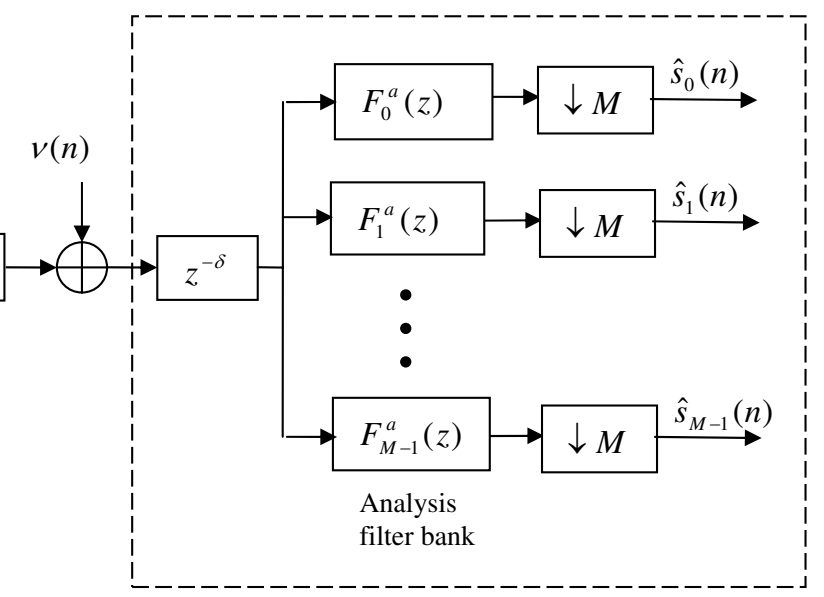

Fig. 1. Block schematic of a CMFB-based transmultiplexer.

Let $Q_{0}^{\mathrm{a}}(z), Q_{1}^{\mathrm{a}}(z), \cdots, Q_{2 M-1}^{\mathrm{a}}(z)$ denote the transfer functions between the input $x(n)$ and the analyzed outputs $u_{0}^{o}(n), u_{1}^{o}(n), \cdots, u_{2 M-1}^{o}(n)$, respectively. We recall from the theory of CMFB that $Q_{k}^{\mathrm{a}}(z)=d_{k} P_{0}\left(z W_{2 M}^{k+0.5}\right)$ for $k=0,1, \cdots, 2 M-1,[28]$. The CMFB analysis filters are generated by adding the pairs of $Q_{k}^{\mathrm{a}}(z)$ and $Q_{2 M-1-k}^{\mathrm{a}}(z)$, for $k=0,1, \cdots, M-1$. This leads to $M$ analysis filters [28]

$$
F_{k}^{\mathrm{a}}(z)=Q_{k}^{\mathrm{a}}(z)+Q_{2 M-1-k}^{\mathrm{a}}(z), \quad k=0,1, \cdots, M-1 .
$$

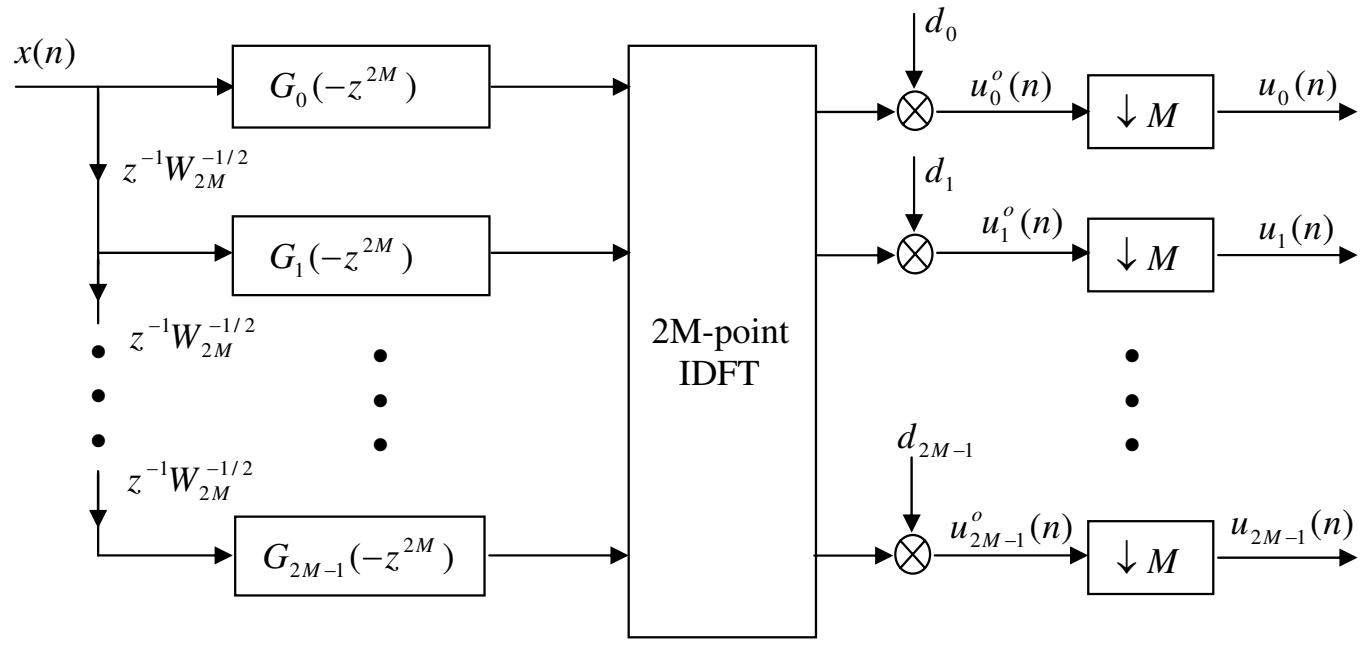

Fig. 2. The analysis CMFB structure that is proposed for CMT.

The synthesis filters, $F_{k}^{\mathrm{S}}(z)$, are given as [28]

$$
F_{k}^{\mathrm{s}}(z)=Q_{k}^{\mathrm{s}}(z)+Q_{2 M-1-k}^{\mathrm{s}}(z), \quad k=0,1, \cdots, M-1
$$

where $Q_{k}^{\mathrm{s}}(z)=z^{-N} Q_{k, *}^{\mathrm{a}}\left(z^{-1}\right)$ and the subscript $*$ means conjugating the coefficients.

In a CMT transceiver, the synthesis filters $F_{k}^{\mathrm{S}}(z)$ are used at the transmitter. However, at the receiver we resort to using the complex coefficient analysis filters $Q_{k}^{\mathrm{a}}(z)$. In the absence of channel, and assuming that a pair of synthesis and analysis CMFB with PR are used, we get [28]

$$
u_{k}(n)=\frac{1}{2}\left[s_{k}(n-\Delta)+j r_{k}(n)\right]
$$


where $r_{k}(n)$ arises because of ISI from the $k$ th sub-channel and ICI from other sub-channels. The PR property of CMFB allows us to remove the ISI plus ICI term $r_{k}(n)$ and extract the desired symbol $s_{k}(n-\Delta)$ simply by taking twice of the real part of $u_{k}(n)$. This, of course, is in the absence of channel. The presence of channel affects $u_{k}(n)$, and $s_{k}(n-\Delta)$ can no longer be extracted by the above procedure.

In order to include the effect of the channel, we make the simplifying, but reasonable, assumption that the number of sub-bands is sufficiently large such that the channel frequency response, $H(z)$, over the $k$ th sub-channel can be approximated by a complex constant gain $h_{k}$. Moreover, we assume that variation of the channel group delay over the band of transmission is negligible. Then, in the presence of channel, we obtain

$$
u_{k}(n) \approx \frac{1}{2}\left[s_{k}(n-\Delta)+j r_{k}(n)\right] \times h_{k}+\nu_{k}(n)
$$

where $\nu_{k}(n)$ is the channel additive noise after filtering. The numerical results presented in Section VI show that for a reasonly large value of $M$, the assumptions of flat channel gain over each subcarrier is very reasonable. However, for channels with bridged taps the group delay variation may not be insignificant. Nevertheless, the incurred performance loss, found through simulation, is tolerable. Clearly, the latter loss could be compensated by adjusting the delay in each subcarrier channel separately. But, this would be at the cost of significant increase in the receiver complexity which may not be justifiable for such a minor improvement.

Considering (5), an estimate of $s_{k}(n)$ can be obtained as follows:

$$
\begin{aligned}
\hat{s}_{k}(n) & =\Re\left\{w_{k}^{*} u_{k}(n)\right\} \\
& =w_{k, \mathrm{R}} u_{k, \mathrm{R}}(n)+w_{k, \mathrm{I}} u_{k, \mathrm{I}}(n)
\end{aligned}
$$

where the subscripts $\mathrm{R}$ and I denote the real and imaginary parts of the respective variables. Equation (6) shows that the distorted received signal $u_{k}(n)$ can be equalized by using a complex tap weight $w_{k}^{*}$ or, equivalently, by using two real tap weights $w_{k, \mathrm{R}}$ and $w_{k, \mathrm{I}}$. If we define the optimum value of $w_{k}^{*}, w_{k, \text { opt }}^{*}$, as the one that maximizes the signal-to-noise plus interference ratio at the equalizer output, we find that

$$
w_{k, \mathrm{opt}}^{*}=\frac{2}{h_{k}} .
$$

At this point, we shall make some comments about DWMT and clarify the difference between the proposed receiver and that of the DWMT [22]. In DWMT, the analyzed sub-carrier signals that are passed to the post-combiner equalizers are the outputs of $F_{k}^{\mathrm{a}}(z)$ filters, i.e., $2 \Re\left\{u_{k}(n)\right\}$. Since these outputs are real-valued, they lack the channel phase information and, hence, a transversal equalizer with input $2 \Re\left\{u_{k}(n)\right\}$ will short fall in removing ISI and ICI. To compensate for the loss of phase information, in DWMT, it was proposed that samples of signals from $k$ th sub-carrier channel and its adjacent sub-carrier channels be combined together for equalization. Theoretical explanation of why this method works can be found in [39]. Hence, the main difference between DWMT and CMT is their respective receiver structures. DWMT uses $F_{k}^{a}(z)$ as analysis filters. CMT, on the other hand, uses the analysis filters $Q_{k}^{a}(z)$. This (minor) change in the receiver allows CMT to adopt simple equalizers with only two real valued tap weights per sub-carrier band while DWMT needs equalizers that are an order of magnitude higher in complexity.

\section{iII. Efficient Realization of Analysis CMFB}

Efficient implementation of synthesis CMFB using discrete cosine transform (DCT) can be found in [28]. This will be used at the transmitter side of a CMT transceiver. At the receiver, as discussed above, we use a modified structure of analysis CMFB. Thus, efficient implementations that are available for the conventional analysis CMFB, e.g., [28], are of no use here. We develop a computationally efficient realization of the analysis CMFB by modifying the structure of Fig. 2. 
At the receiver, we need to implement filters $Q_{0}^{\mathrm{a}}(z), Q_{1}^{\mathrm{a}}(z), \cdots, Q_{M-1}^{\mathrm{a}}(z)$. Recalling that $Q_{2 M-1-k}^{\mathrm{a}}\left(e^{-j \omega}\right)=\left[Q_{k}^{\mathrm{a}}\left(e^{j \omega}\right)\right]^{*}$ and $x(n)$ is real-valued, we argue that these filters can equivalently be implemented by realizing $Q_{k}^{\mathrm{a}}(z)$ for $k=0,2,4, \cdots, 2 M-2$, i.e., for even values of $k$ only; $Q_{1}^{\mathrm{a}}(z)$, for instance, is realized by taking the conjugate of the output of $Q_{2 M-2}^{\mathrm{a}}(z)$. We thus note from Fig. 2 that

$$
\begin{aligned}
Q_{2 k}^{\mathrm{a}}(z) & =d_{2 k} \sum_{l=0}^{2 M-1}\left(z^{-1} W_{2 M}^{-1 / 2}\right)^{l} G_{l}\left(-z^{2 M}\right) W_{2 M}^{-2 k l} \\
& =d_{2 k} \sum_{l=0}^{M-1}\left[z^{-l}\left(G_{l}\left(-z^{2 M}\right)+j z^{-M} G_{l+M}\left(-z^{2 M}\right)\right) W_{2 M}^{-l / 2}\right] W_{M}^{-k l}
\end{aligned}
$$

Using (8) to modify Fig. 2 and using the noble identities, [28], to move the decimators to the position before the polyphase component filters, we obtain the efficient implementation of Fig. 3. This implementation has a computational complexity that is approximately one half of that of the original structure in Fig. 2, assuming the the decimators in the latter are also moved the position before the polyphase component filters - here, the $2 M$-point IDFT in Fig. 2 is replaced by an $M$-point IDFT. The block $C$ is to reorder and conjugate the output samples, wherever needed.

The realization of Fig. 3 involves implementation of $M$ polyphase component filters $G_{l}\left(-z^{2}\right)+$ $j z^{-1} G_{l+M}\left(-z^{2}\right), M$ complex scaling factors $W_{2 M}^{-l / 2}$, an $M$-point IDFT, and the group delay compensatory coefficients $d_{l}$. The latter coefficients may be deleted as they can be lumped together with the equalizer coefficients $w_{k}^{*}$.

The structure of Fig. 3 should be compared with the analysis CMFB/SMFB structure of [43]. On the basis of the operation count (the number of multiplications and additions per unit of time) the two structures are similar. However, they are different in their structural details. While Fig. 3 uses an $M$-point IDFT with complex-valued inputs, the CMFB/SMFB structure uses two separate transforms (a DCT and a DST) with real-valued inputs. Therefore, a preference of one against the other depends on the available hardware or software platform on which the system is to be implemented.

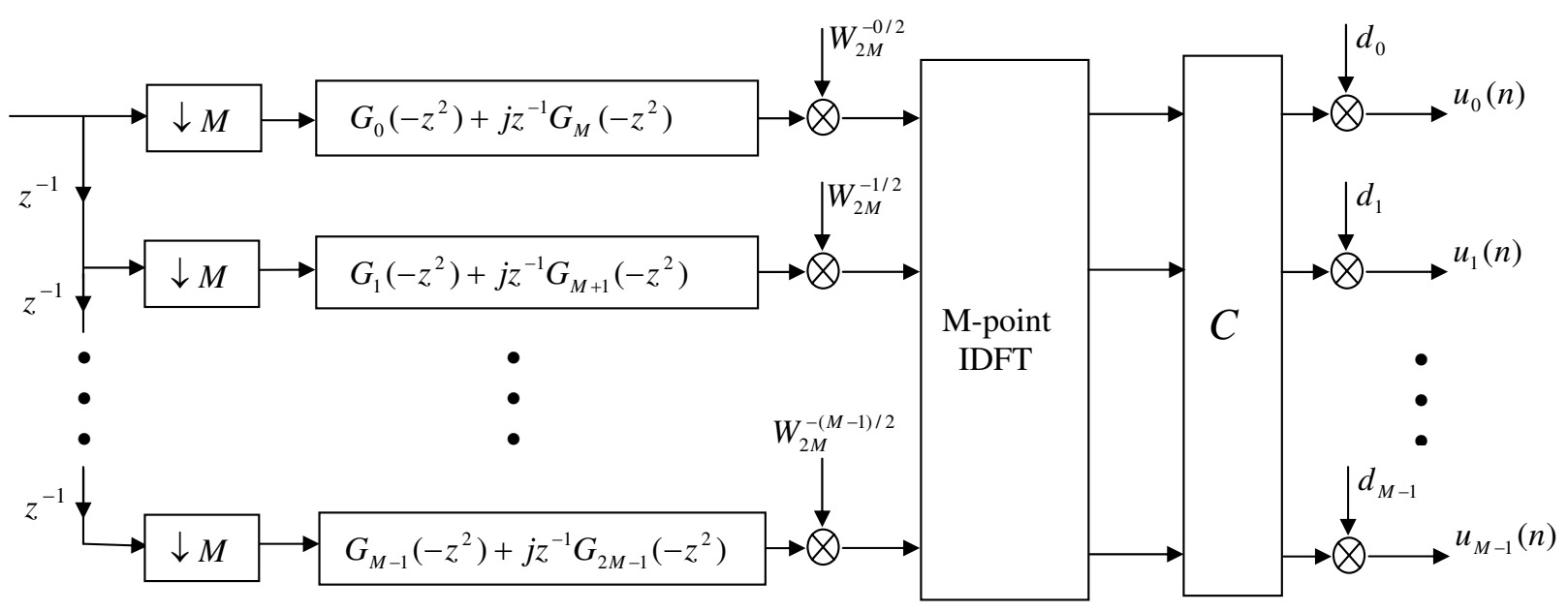

Fig. 3. Efficient implementation of the analysis CMFB.

\section{Prototype Filter Design}

Prototype filter design is an important issue in CMT modulation. In CMFB, conventionally, the prototype filter is designed to satisfy the PR property. However, in the application of interest to this paper, the presence of channel results in a loss of the PR property. In this section, we take 
note of this fact and propose a new prototype filter design scheme which instead of designing for PR aims at minimizing the ISI plus ICI and maximizing the stopband attenuation. We thus adopt an NPR design. For this purpose, we develop a cost function in which a balance between the ISI plus ICI and the stopband attenuation is struck through a design parameter. A similar approach was adopted in [32] for designing prototype filter in FMT.

\section{A. ISI and ICI}

Referring to Figs. 1 and 2, and assuming that only adjacent sub-channels overlap, in the absence of channel noise, we obtain

$$
U_{k}^{o}(z)=z^{-\delta}\left(S_{k}\left(z^{M}\right) F_{k}^{\mathrm{s}}(z)+S_{k-1}\left(z^{M}\right) F_{k-1}^{\mathrm{s}}(z)+S_{k+1}\left(z^{M}\right) F_{k+1}^{\mathrm{s}}(z)\right) H(z) Q_{k}^{\mathrm{a}}(z),
$$

where $S_{k}(z)$ is the z-transform of $s_{k}(n)$ and z-transforms of other sequences are defined similarly. Substituting (3) in (9) and noting that for $k \neq 0$ and $M-1, Q_{k}^{\text {a }}(z)$ has no (significant) overlap with $Q_{2 M-k}^{\mathrm{s}}(z), Q_{2 M-1-k}^{\mathrm{s}}(z)$ and $Q_{2 M-2-k}^{\mathrm{s}}(z)$, we obtain, for ${ }^{1} k \neq 0$ and $M-1$,

$$
U_{k}^{o}(z)=z^{-\delta}\left(S_{k}\left(z^{M}\right) Q_{k}^{\mathrm{s}}(z)+S_{k-1}\left(z^{M}\right) Q_{k-1}^{\mathrm{s}}(z)+S_{k+1}\left(z^{M}\right) Q_{k+1}^{\mathrm{s}}(z)\right) H(z) Q_{k}^{\mathrm{a}}(z) .
$$

We use the notation $[\cdot]_{\downarrow}$ to denote the $M$-fold decimation. Recalling that $\left[U_{k}^{o}(z)\right]_{\downarrow M}=U_{k}(z)$ and for arbitrary functions $X(z)$ and $Y(z),\left[X\left(z^{M}\right) Y(z)\right]_{\downarrow M}=X(z)[Y(z)]_{\downarrow M}$, from (10), we obtain

$$
\begin{aligned}
U_{k}(z)=S_{k}(z)\left[z^{-\delta} Q_{k}^{\mathrm{s}}(z) H(z) Q_{k}^{\mathrm{a}}(z)\right]_{\downarrow M} & +S_{k-1}(z)\left[z^{-\delta} Q_{k-1}^{\mathrm{s}}(z) H(z) Q_{k}^{\mathrm{a}}(z)\right]_{\downarrow M} \\
& +S_{k+1}(z)\left[z^{-\delta} Q_{k+1}^{\mathrm{s}}(z) H(z) Q_{k}^{\mathrm{a}}(z)\right]_{\downarrow M} .
\end{aligned}
$$

Using (7), we get the estimate of $S_{k}(z)$ (the equalized signal) as

$$
\begin{aligned}
\hat{S}_{k}(z) & =\Re\left\{\frac{2}{h_{k}} U_{k}(z)\right\} \\
& =S_{k}(z) A_{k}(z)+S_{k-1}(z) B_{k}(z)+S_{k+1}(z) C_{k}(z)
\end{aligned}
$$

where

$$
\begin{aligned}
A_{k}(z) & =\Re\left\{\frac{2}{h_{k}}\left[z^{-\delta} Q_{k}^{\mathrm{s}}(z) H(z) Q_{k}^{\mathrm{a}}(z)\right]_{\downarrow M}\right\} \\
B_{k}(z) & =\Re\left\{\frac{2}{h_{k}}\left[z^{-\delta} Q_{k-1}^{\mathrm{s}}(z) H(z) Q_{k}^{\mathrm{a}}(z)\right]_{\downarrow M}\right\} \\
C_{k}(z) & =\Re\left\{\frac{2}{h_{k}}\left[z^{-\delta} Q_{k+1}^{\mathrm{s}}(z) H(z) Q_{k}^{\mathrm{a}}(z)\right]_{\downarrow M}\right\}
\end{aligned}
$$

and $\Re\{\cdot\}$ when applied to a transfer function means forming a transfer function by taking the real parts of the coefficients of the argument. When applied to a complex number of vector, $\Re\{\cdot\}$ denotes the real part of.

If the prototype filter was designed to satisfy the PR condition, in the absence of the channel, we would have $A_{k}(z)=z^{-\Delta}, B_{k}(z)=0$ and $C_{k}(z)=0$. In the presence of the channel, these properties are lost and accordingly the ISI and ICI powers at $k$ th sub-channel are expressed, respectively, as

$$
\zeta_{k, \text { ISI }}=\left(\mathbf{a}_{k}-\mathbf{u}\right)^{\mathrm{T}}\left(\mathbf{a}_{k}-\mathbf{u}\right)
$$

and

$$
\zeta_{k, \mathrm{ICI}}=\mathbf{b}_{k}^{\mathrm{T}} \mathbf{b}_{k}+\mathbf{c}_{k}^{\mathrm{T}} \mathbf{c}_{k}
$$

\footnotetext{
${ }^{1}$ In DSL applications, the sub-channels near origin $(k=0)$ and $\pi(k=M-1)$ do not carry any data [22].
} 
where $\mathbf{a}_{k}, \mathbf{b}_{k}$ and $\mathbf{c}_{k}$ are the column vectors of the coefficients of $A_{k}(z), B_{k}(z)$ and $C_{k}(z)$, respectively, and $\mathbf{u}$ is a column vector with $\Delta$ th element of 1 and 0 elsewhere.

The above results were given for the case when only the adjacent bands overlap. When each sub-carrier band overlaps with more than two of its neighbor sub-carrier bands, the above results may be easily extended by defining more polynomials like $B_{k}(z)$ and $C_{k}(z)$ and accordingly adding more terms to (17).

\section{B. The Cost Function}

The cost function that we minimize for designing the prototype filter is defined as

$$
\zeta=\zeta_{s}+\gamma\left(\zeta_{\mathrm{ISI}}+\zeta_{\mathrm{ICI}}\right)
$$

where $\zeta_{s}$ is the stopband energy of the prototype filter, defined below, and $\gamma$ is a positive parameter which should be selected to strike a balance between the stopband energy and ISI plus ICI. A larger $\gamma$ leads to a smaller ISI plus ICI. Here and in the remaining discussions, for convenience, we drop the sub-carrier band index $k$ of $\zeta_{k, \text { ISI }}$ and $\zeta_{k, \text { ICI }}$.

Selecting the frequency grid $\left\{\omega_{0}, \omega_{1}, \cdots, \omega_{L-1}\right\}$ in the interval $\left[\omega_{\mathrm{s}}, \pi\right]$, where $\omega_{\mathrm{s}}$ is the stopband edge of the prototype filter, we define

$$
\zeta_{s}=\frac{1}{L} \sum_{l=0}^{L-1}\left|P\left(e^{j \omega_{l}}\right)\right|^{2} .
$$

We also assume that the prototype filter, $P(z)$, has a length of $2 m M$. This choice of the length follows that of the PR CMFB [28], and is believed to be appropriate since here we design a filter bank with NPR property. Moreover, we follow the PR CMFB convention and design a linear phase prototype filter. This implies that

$$
P\left(e^{j \omega_{l}}\right)=e^{-j \omega_{l}(m M-0.5)} \sum_{n=0}^{m M-1} 2 p(m M+n) \cos \left(\omega_{l}(n+0.5)\right),
$$

where $p(n)$ is the $n$th coefficient of $P(z)$. Rearranging (20), we obtain

$$
\mathbf{C p}=\left[\begin{array}{c}
e^{j \omega_{0}(m M-0.5)} P\left(e^{j \omega_{0}}\right) \\
e^{j \omega_{1}(m M-0.5)} P\left(e^{j \omega_{1}}\right) \\
\vdots \\
e^{j \omega_{L-1}(m M-0.5)} P\left(e^{j \omega_{L-1}}\right)
\end{array}\right]
$$

where $\mathbf{C}$ is an $L \times m M$ matrix with the $i j$ th element of $c_{i, j}=2 \cos \left(\omega_{i-1}(j-0.5)\right)$ and $\mathbf{p}=$ $[p(m M) p(m M+1) \cdots p(2 m M-1)]^{\mathrm{T}}$. Using (21), (19) may be rearranged as

$$
\zeta_{s}=\frac{1}{L} \mathbf{p}^{\mathrm{T}} \mathbf{C}^{\mathrm{T}} \mathbf{C} \mathbf{p}
$$

To calculate $\zeta_{\mathrm{ISI}}$ and $\zeta_{\mathrm{ICI}}$, we note that since $Q_{k}^{\mathrm{s}}(z) Q_{k}^{\mathrm{a}}(z), Q_{k-1}^{\mathrm{s}}(z) Q_{k}^{\mathrm{a}}(z)$ and $Q_{k+1}^{\mathrm{s}}(z) Q_{k}^{\mathrm{a}}(z)$ are narrowband filters centered around the $k$ th subcarrier band and over this band $H(z)$ may be approximated by the constant gain $h_{k}$, from (13)-(15), we obtain

$$
\begin{aligned}
\mathbf{a}_{k} & =2 \Re\left\{\left[\mathbf{q}_{k}^{\mathrm{s}} \star \mathbf{q}_{k}^{\mathrm{a}}\right]_{\downarrow M}\right\} \\
\mathbf{b}_{k} & =2 \Re\left\{\left[\mathbf{q}_{k-1}^{\mathrm{s}} \star \mathbf{q}_{k}^{\mathrm{a}}\right]_{\downarrow M}\right\} \\
\mathbf{c}_{k} & =2 \Re\left\{\left[\mathbf{q}_{k+1}^{\mathrm{s}} \star \mathbf{q}_{k}^{\mathrm{a}}\right]_{\downarrow M}\right\}
\end{aligned}
$$

where $\star$ stands for convolution and $\mathbf{q}_{k}^{\mathrm{s}}$ and $\mathbf{q}_{k}^{\mathrm{a}}$ are the column vectors of coefficients of $z^{-\delta} Q_{k}^{\mathrm{s}}(z)$ and $Q_{k}^{\mathrm{a}}(z)$, respectively. 
Equation (23) may be expressed in a matrix form as

$$
\mathbf{a}_{k}=2 \Re\left\{\mathbf{Q q}_{k}^{\mathrm{a}}\right\}
$$

where the matrix $\mathbf{Q}$ is obtained by arranging $\mathbf{q}_{k}^{\mathrm{s}}$ and its shifted copies in a matrix $\mathbf{Q}^{o}$ and decimating $\mathbf{Q}^{o}$ by $M$ in each column. Noting that $q_{k}^{\mathrm{a}}(n)=p(n) e^{j\left(\frac{\pi}{M}(k+0.5)\left(n-\frac{N}{2}\right)+(-1)^{k} \frac{\pi}{4}\right)}, p(n)=$ $p(2 m M-n-1)$, and defining $\mathbf{D}$ as a diagonal matrix with the $n$th diagonal element $d_{n, n}=$ $e^{j\left(\frac{\pi}{M}(k+0.5)\left(n-\frac{N}{2}\right)+(-1)^{k} \frac{\pi}{4}\right)},(26)$ may be written as

$$
\mathbf{a}_{k}=2 \Re\{\mathbf{Q D}\}\left[\begin{array}{l}
\mathbf{p}^{\mathrm{r}} \\
\mathbf{p}
\end{array}\right]
$$

where $\mathbf{p}^{\mathrm{r}}$ is obtained by reversing the order of elements of $\mathbf{p}$. In matrix/vector notations $\mathbf{p}^{\mathbf{r}}=\mathbf{J} \mathbf{p}$ where $\mathbf{J}$ is the antidiagonal matrix with the antidiagonal elements of 1 . Using this in (27), we obtain

$$
\mathbf{a}_{k}=\mathbf{E} \mathbf{p}
$$

where $\mathbf{E}=2 \Re\{\mathbf{Q D}\}\left[\begin{array}{c}\mathbf{J} \\ \mathbf{I}\end{array}\right]$ and $\mathbf{I}$ is the identity matrix. Substituting (28) in (16), we obtain

$$
\zeta_{\text {ISI }}=(\mathbf{E p}-\mathbf{u})^{\mathrm{T}}(\mathbf{E} \mathbf{p}-\mathbf{u})
$$

Following similar steps, we obtain

$$
\zeta_{\text {ICI }}=\mathbf{p}^{\mathrm{T}} \mathbf{F}^{\mathrm{T}} \mathbf{F} \mathbf{p}
$$

where the matrix $\mathbf{F}$ is constructed in the same way as $\mathbf{E}$, by replacing $\mathbf{q}_{k}^{\mathrm{s}}$ with $\left[\begin{array}{c}\mathbf{q}_{k-1}^{\mathrm{s}} \\ \mathbf{q}_{k+1}^{\mathrm{s}}\end{array}\right]$.

Now substituting (22), (29) and (30) in (18), we obtain

$$
\zeta=(\mathbf{G p}-\mathbf{v})^{\mathrm{T}}(\mathbf{G} \mathbf{p}-\mathbf{v}),
$$

where $\mathbf{G}=\left[\begin{array}{c}\mathbf{E} \\ \mathbf{F} \\ \frac{1}{\sqrt{\gamma}} \mathbf{C}\end{array}\right], \mathbf{v}=\left[\begin{array}{l}\mathbf{u} \\ \mathbf{0}\end{array}\right]$, and $\mathbf{0}$ is a zero column vector with proper length.

\section{Minimization of the Cost Function}

We note that $\mathbf{q}_{k}^{\mathrm{s}}$ and thus $\mathbf{G}$ depends on $\mathbf{p}$. Hence, the cost function (31) is fourth order in the filter coefficients, $p(n)$, and thus its minimization is non-trivial. Rossi et al [31] proposed an iterative least squares (ILS) minimization for a similar problem. They formulated the same filter design problem for the case of a PR CMFB. Adopting the method of Rossi et al [31], we minimize $\zeta$ by using the following procedure:

Step 1: Let $\mathbf{p}=\mathbf{p}_{0}$; an initial choice.

Step 2: Construct the matrix $\mathbf{G}$ using the current value of $\mathbf{p}$.

Step 3: Form the normal equation $\mathbf{\Psi} \mathbf{p}=\boldsymbol{\theta}$, where $\boldsymbol{\Psi}=\mathbf{G}^{\mathrm{T}} \mathbf{G}$ and $\boldsymbol{\theta}=\mathbf{G}^{\mathrm{T}} \mathbf{v}$.

Step 4: Compute $\mathbf{p}_{1}=\boldsymbol{\Psi}^{-1} \boldsymbol{\theta}$.

Step 5: $\frac{\mathbf{p}_{0}+\mathbf{p}_{1}}{2} \rightarrow \mathbf{p}_{0}$ and go back to Step 2 .

Steps 2 to 5 are run for sufficient iterations until the design converges.

Numerical examples show that this algorithm can converge to a good design if the initial choice $\mathbf{p}=\mathbf{p}_{0}$, and the parameter $\gamma$ are selected properly. Compared to other CMFB prototype filter designs, this method is attractive because of its relatively low computational complexity. Other methods, such those based on paraunitary property of PR filter banks [28] are too complicated and hard to apply to filter banks with large number of sub-bands; the case of interest in this 
paper. Beside, such design methods are not useful here because we are not interested in designing filter banks with PR property. Because of these reasons, we found the approach of [31] the most appropriate in this paper and thus elaborate on it further.

In CMT, we are interested in very long prototype filters whose length exceeds a few thousands. This means in the normal equation $\Psi \mathbf{p}=\boldsymbol{\theta}, \boldsymbol{\Psi}$ is a very large matrix. Hence Step 4 in the above procedure may be computationally expensive and sensitive to numerical errors. In our experiments where we designed filters with length of up to 3072, using the MATLAB routine of [31] we did not encounter any numerical inaccuracy problem. However, the design times were excessively long. Since we wished to design many prototype filters, we had to find other alternative methods that could run faster. Fortunately, we found the Gauss-Seidel method as a good alternative.

Gauss-Seidel method is a general mathematical optimization method that is applicable to variety of optimization problems [47], [48]. It finds the optimum parameters of interest by adopting an iterative approach. A cost function is chosen and it is optimized by successively optimizing one of the cost function parameters at a time, while other parameters are fixed. A particular version of Gauss-Seidel reported in [49] can be used to minimize the difference $\mathbf{G p}-\mathbf{v}$ in the least squares sense without resorting to the normal equation $\boldsymbol{\Psi} \mathbf{p}=\boldsymbol{\theta}$. Moreover, an accelerated step that improves the convergence rate of the Gauss-Seidel method has been proposed in [49]. Through numerical examples, we found that the accelerated Gauss-Seidel method could be used to replace for Step 4 in the above procedure, with the advantage of speeding up the design time by an order of magnitude or more.

Here, we request that the interested readers refer to [49] for details of the accelerated Gauss-Seidel method. In an Appendix at the end of this paper, we have given the script of a MATLAB m-file that we have used for the design of the prototype filters. The prototype filter that we have used to generate the simulation results of Section VI is based on the following parameters: $M=512$, $m=3, f_{\mathrm{s}}=\frac{1.2}{2 M}, \gamma=100, K=2$.

\section{Computational Complexity and Latency}

Computational complexity and latency are two issues of concern in any system implementation. In this section, we present a detailed evaluation of the computational complexity and latency of CMT and compare that against z-DMT and FMT.

\section{A. Computational Complexity}

The computational blocks involved in z-DMT and their associated operation counts are summarized in Table I. The number of operations given for each block are based on some of the best available algorithms. In particular, we have considered using the split radix FFT algorithm [29] for implementation of the modulator and demodulator blocks. We have counted each complex multiplication as three real multiplications and three real additions [29]. The variable $M$, here, indicates the number of sub-carriers in z-DMT. The FEQs are single tap complex equalizers used to equalize the demodulated data symbols. We have not accounted for possible adaptation of the equalizers. The RFI cancellation also is not accounted for, as it varies with the number of interferers. For instance, when there is no RFI, the computational load introduced by the canceller is limited to channel sounding for detection of RFI and this can be negligible. On the other hand, when an RFI is detected the system may momentarily have to take a relatively large computational load to setup the canceller parameters. Thus, the issue here might be that of a peak computational power load. Since accounting for this can complicate our analysis, we simply ignore the complexity imposed by the RFI canceller and only comment that this can be a burden to a practical z-DMT system.

Table II lists the computational blocks of a CMT transceiver and the number of operations for each block. Here, the modulator and demodulator are the CMFB synthesis and analysis filter banks, respectively. The operation counts of modulation are based on the efficient implementation of synthesis CMFB with DCT in [28], and the operation counts of demodulation are based on Fig. 3. 
TABLE I

SUMMARY OF COMPUTATIONAL COMPLEXITY OF Z-DMT TRANSCEIVER.

\begin{tabular}{rcc}
\hline \hline & Additions & Multiplications \\
\hline Modulator (IFFT) & $M\left(3 \log _{2} M-2\right)$ & $M\left(\log _{2} M-2\right)$ \\
Demodulator (FFT) & $M\left(3 \log _{2} M-2\right)$ & $M\left(\log _{2} M-2\right)$ \\
FEQ & $3 M$ & $3 M$ \\
\hline \hline
\end{tabular}

TABLE II

SUMMARY OF COMPUTATIONAL COMPLEXITY OF CMT TRANSCEIVER.

\begin{tabular}{rcc}
\hline \hline & Additions & Multiplications \\
\hline Modulator & $M\left(1.5 \log _{2} M+2 m\right)$ & $M\left(0.5 \log _{2} M+2 m+1\right)$ \\
Demodulator & $M\left(3 \log _{2} M+2 m-2\right)$ & $M\left(\log _{2} M+2 m\right)$ \\
Equalizer & $M$ & $2 M$ \\
\hline \hline
\end{tabular}

Two-tap equalizers, discussed in Section II, are used to mitigate ISI and ICI at the demodulator outputs. Here also we have not accounted for possible adaptation of the equalizers. The $d_{k}$ coefficients at the output of the analysis CMFB of Fig. 3 are not accounted for as they can be combined with the equalizers. The parameters appeared in Table II are the number of sub-carriers, $M$, and the overlapping factor, $m$; the length of prototype filter $P(z)$ is $2 m M$.

Table III lists the computational blocks of a FMT transceiver and the number of operations for each block. The operation counts are based on the efficient realization in [36]. Similar to z-DMT and CMT, here also, the adaptation of the equalizer coefficients is not counted. $M$ is the number of sub-carrier channels. The prototype filter length is $2 m M . N_{\mathrm{f}}$ and $N_{\mathrm{b}}$ denote the number of taps in the feedforward and feedback sections of DFE, respectively.

Adding up the number of operations given in each of Tables I, II and III, and normalizing the results by the block length ( $2 M$ for z-DMT and FMT, and $M$ for CMT), the per sample complexity of z-DMT, CMT and FMT are obtained as

$$
\begin{aligned}
C_{\mathrm{DMT}} & =4 \log _{2} M-1 \\
C_{\mathrm{CMT}} & =6 \log _{2} M+8 m+2 \\
C_{\mathrm{FMT}} & =4 \log _{2} M+4 m+4\left(N_{\mathrm{f}}+N_{\mathrm{b}}\right)-7
\end{aligned}
$$

For all comparisons in this paper, the following parameters are used. For z-DMT, we choose $M=2048$. This is consistent with the VDSL draft standard [37] and the latest reports on z-DMT [15]. For FMT, we follow [36] and choose $M=128, m=10, N_{\mathrm{f}}=26$ and $N_{\mathrm{b}}=9$. For CMT, we experimentally found that $M=512$ and $m=3$ are sufficient to get very close to the best results that it can achieve. With these choices, we obtain $C_{\mathrm{DMT}}=43, C_{\mathrm{CMT}}=80$ and $C_{\mathrm{FMT}}=201$ operations per sample. It is noted that FMT is significantly more complex than z-DMT and CMT, and the computational complexity of CMT is about 2 times that of the z-DMT. However, we

\section{TABLE III}

SumMARY OF COMPUTATIONAL COMPLEXITY OF FMT TRANSCEIVER.

\begin{tabular}{rcc}
\hline \hline & Additions & Multiplications \\
\hline Modulator & $M\left(3 \log _{2} M+2 m-4\right)$ & $M\left(\log _{2} M+2 m-2\right)$ \\
Demodulator & $M\left(3 \log _{2} M+2 m-4\right)$ & $M\left(\log _{2} M+2 m-2\right)$ \\
Equalizer & $M\left(5 N_{\mathrm{f}}+5 N_{\mathrm{b}}-2\right)$ & $3 M\left(N_{\mathrm{f}}+N_{\mathrm{b}}\right)$ \\
\hline \hline
\end{tabular}


should note that the complexity of z-DMT given here does not include the RFI canceller which, as noted above, can momentarily exhibit a significant computational peak load, whenever a new RFI is detected.

\section{B. Latency}

In the context of our discussion in this paper, the latency is defined as the time delay that each coded information symbol will undergo in passing through a transceiver. In z-DMT, the following operations have to be counted for. A block of data symbols have to be collected in an input buffer before being passed to the modulator. This, which we refer to as buffering delay, introduces a delay equivalent to one block of DMT. While the next block of data symbols are being buffered, the modulator processes the previous block of data. This introduces another block of DMT delay. We refer to this as processing delay. The buffering and processing delay together count for a delay of equivalent to two blocks of DMT at the transmitter. Following the same discussion, we find that the receiver also introduces two blocks of DMT delay. Thus, the total latency introduced by the transmitter and receiver in z-DMT (or DMT, in general) is given by

$$
\Delta_{\mathrm{DMT}}=4 T_{\mathrm{DMT}}
$$

where $T_{\mathrm{DMT}}$ is the time duration of each z-DMT block. This includes a block of data and the associated cyclic extensions. We also note that the channel introduces some delay. Since this delay is small and common to the three schemes, we ignore it in all the latency calculations. We thus use the following approximation for the purpose of comparisons

$$
\Delta_{\mathrm{DMT}}=4\left(2 M+\mu_{\mathrm{cp}}+\mu_{\mathrm{cs}}\right) T_{\mathrm{s}}
$$

where $\mu_{\mathrm{cp}}$ and $\mu_{\mathrm{cs}}$ are the length of cyclic prefix and cyclic suffix, respectively, and $T_{\mathrm{s}}$ is the sampling interval which in the case of VDSL is $0.0453 \mu \mathrm{s}$, corresponding to the sampling frequency of $22.08 \mathrm{MHz}$.

The latency calculation of CMT is straightforward. The delay introduced by the synthesis and analysis filter banks is determined by the total group delay introduced by them. It is equal to the length of the prototype filter times the sampling interval, $T_{\mathrm{s}}$. This results in a delay of $2 m M T_{\mathrm{s}}$. We should add to this the buffering and processing delays. Since each processing of CMT is performed after collecting a block of $M$ samples, the total buffering plus processing delay in a CMT transceiver is equal to $4 M T_{\mathrm{s}}$. The latency of $\mathrm{CMT}$ is thus obtained as

$$
\Delta_{\mathrm{CMT}}=(2 m+4) M T_{\mathrm{s}}
$$

The latency calculation of FMT is similar to that of CMT. Delays are introduced by the synthesis filter bank, the analysis filter bank and the DFEs. The delay introduced by synthesis and analysis filter banks is $2 m M T_{\mathrm{s}}$. A total buffering and processing delay $4 M T_{\mathrm{s}}$ should be added to this. The delay introduced by the feed-forward section of DFE is $N_{\mathrm{f}} / 2$ samples. Since fractional space DFEs work at the rate decimated by $M$, the introduced delay is $M N_{\mathrm{f}} T_{\mathrm{s}} / 2$. The latency of FMT is thus

$$
\Delta_{\mathrm{FMT}}=\left(2 m+8+\frac{N_{\mathrm{f}}}{2}\right) M T_{\mathrm{s}} .
$$

As noted in section V-A, we choose $M=2048$ and $\mu_{\mathrm{cp}}+\mu_{\mathrm{cs}}=320$ for z-DMT, $M=512$ and $m=3$ for CMT, and choose $M=128, m=10, N_{\mathrm{f}}=26$ and $N_{\mathrm{b}}=9$ for FMT. These result in the latency values $\Delta_{\mathrm{DMT}}=800 \mu \mathrm{s}, \Delta_{\mathrm{CMT}}=232 \mu \mathrm{s}$ and $\Delta_{\mathrm{FMT}}=238 \mu \mathrm{s}$. We note that the latencies of CMT and FMT are significantly lower than that of z-DMT. This, clearly, is because of the use of a much smaller block size, $M$, in CMT and FMT. 


\section{Vi. Simulation Results and Discussion}

The system model used for simulations is presented in Fig. 4. This setup accommodates NEXT (near-end cross-talk) and FEXT (far-end cross-talk) coupling, background noise and RFI ingress. The setup assumes that the system is in training mode and thus transmitted symbols are available at the receiver. Hence, we can measure SNRs at various sub-carrier bands and accordingly find the corresponding bit allocations. The symbol generator output is 4-QAM in the case of z-DMT and FMT, and antipodal binary for CMT.

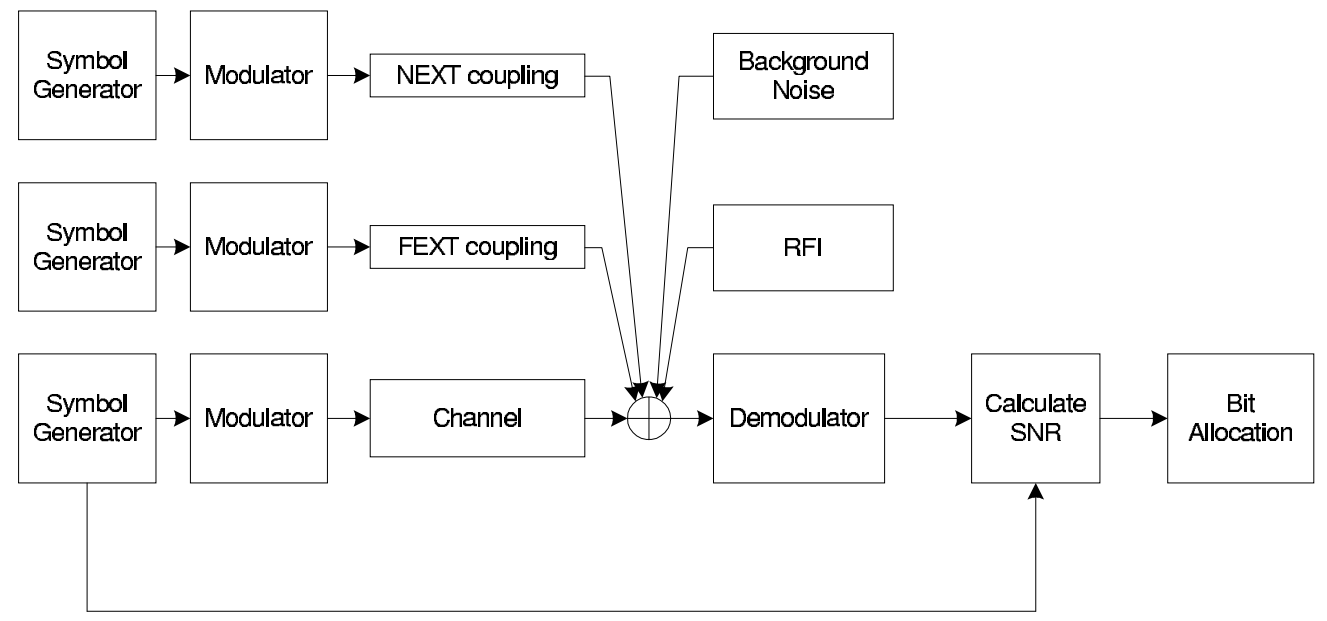

Fig. 4. Simulation setup.

To make comparisons with the previous works possible, we follow simulation parameters of [15], as close as possible. We use a transmission bandwidth of $300 \mathrm{kHz}$ to $11 \mathrm{MHz}$. The noise sources include a mix of ETSI'A', [50], 25 NEXT and 25 FEXT disturbers. Transmit band allocation is also performed according to [15].

\section{A. System Parameters}

The number of sub-carriers, $M$, and the length of the prototype filter, $2 m M$, are the two most important parameters in CMT. Obviously, the system performance improves as one or both of these parameters increase. However, as we may recall from the results of Section $\mathrm{V}$ both system complexity and latency increase with $M$ and $m$. It is thus desirable to choose $M$ and $m$ to strike a balance between the system performance and complexity. Moreover, for a given pair of $M$ and $m$, the system performance is affected by the choice of the CMFB prototype filter. An important parameter that affects the performance of CMT is the stopband edge of the prototype filter, $\omega_{\mathrm{s}}$. The optimum value of $\omega_{\mathrm{s}}$ is hard to find. On one hand, the choice of a small $\omega_{\mathrm{s}}$ is desirable as it limits the bandwidth of each sub-carrier and makes the assumption of constant channel gain over each sub-band more accurate. On the other hand, a larger choice of $\omega_{\mathrm{s}}$ improves the stopband attenuation of the prototype filter and this in turn reduces the ICI and noise interference from the non-adjacent sub-bands. Moreover, a large value of $\omega_{\mathrm{s}}$ increases RF ingress noise and the NEXT near the frequency band edges. Unfortunately, because of the complexity of the problem and the variety of the parameters that affect the system performance, a good compromised choice of $M \mathrm{~m}$ and $\omega_{s}$ could only be obtained through extensive numerical tests over a wide variety of channel setups. The details of such results will be reported in [51]. Here, we mention the summary of observations that we have had. The choice of $M=512$ was generally found sufficient to satisfy the approximation 'constant channel gain over each sub-band'. With $M=512$, the choices $m=3$ (thus, a prototype filter length of 3072 ) and $\omega_{s}=\frac{1.2 \pi}{M}$ result in a system which behaves very close to the optimum performance, where the optimum performance is that of an ideal system with non-overlapping sub-carrier bands; see Fig. 6, below. 
In our study we also explored the choices of $m=2$ and $m=1$. The results, obviously, were not as good as those of $m=3$, however, for most cases they were still superior to z-DMT and FMT. Here, because of space limitation, we only present results and compare CMT with z-DMT and FMT when in CMT $M=512, m=3$ and $\omega_{\mathrm{s}}=\frac{1.2 \pi}{M}$. Details of other cases will be reported in [51].

For z-DMT, the number of sub-carriers is set equal to 2048, following the VDSL draft standard [37]. As in [15], we have selected the length of CP equal to 100, determined the length of CS according to the channel group delay, and the length of the pulse-shaping and windowing samples are set equal to 140 and 70 , respectively.

Following the parameters of [36], we use an FMT system with $M=128$ sub-channels, and a prototype filter of length $2 m M$, with $m=10$. The excess bandwidth $\alpha$ is set equal to 0.125 . Persubcarrier equalization is performed by employing a Tomlinson-Harashima precoder with $N_{\mathrm{b}}=9$ taps and a $\mathrm{T} / 2$-spaced linear equalizer with $N_{\mathrm{f}}=26$ taps.

\section{B. Crosstalk Dominated Channels}

The DSL environment is crosstalk dominated due to bundling of wire pairs in binder cables. Here, we consider the performance of z-DMT, CMT and FMT when both NEXT and FEXT are present. Since the three modulation schemes are frequency-division duplexed (FDD) systems, NEXT is significant only near the frequency band edges where there is a change in transmit direction. FEXT, on the other hand, affects all the transmit band.

In our simulations, NEXT and FEXT are generated according to the coupling equations provided in [37] for a 50-pair binder cable as

$$
\operatorname{PSD}_{\mathrm{NEXT}}=K_{\mathrm{NEXT}} S_{\mathrm{d}}(f)\left(N_{\mathrm{d}} / 49\right)^{0.6} f^{1.5}
$$

and

$$
\operatorname{PSD}_{\mathrm{FEXT}}=K_{\mathrm{FEXT}} S_{\mathrm{d}}(f)|H(f)|^{2} d\left(N_{\mathrm{d}} / 49\right)^{0.6} f^{2}
$$

where $K_{\mathrm{NEXT}}$ and $K_{\mathrm{FEXT}}$ are constants with values of $8.818 \times 10^{-14}$ and $7.999 \times 10^{-20}$, respectively, $S_{\mathrm{d}}(f)$ is the PSD of a disturber, $N_{\mathrm{d}}$ is the number of disturbers, $H(f)$ is the channel frequency response, and $d$ is the channel length in meters.

Fig. 5 presents SNR curves demonstrating the impact of NEXT in degrading the performance of z-DMT, CMT and FMT. The results correspond to a $810 \mathrm{~m}$ TP1 line. The arrows $\downarrow$ and $\uparrow$ indicate downstream and upstream bands, respectively. The SNR in each subcarrier channel is measured in the time domain by looking at the power of the residual error after subtracting the transmitted symbols. As one would expect there is a significant performance loss in z-DMT at the points where the transmission direction changes. The CMT and FMT, on the other hand, do not show any visible degradation due to NEXT. It is worth noting that the SNR results of z-DMT match closely those reported in [15].

Another observation in Fig. 5 that requires some comments is that although CMT has a lower SNR compared to z-DMT and FMT, it may achieve a higher transmission rate because of higher bandwidth efficiency - no cyclic extensions or excess bandwidth.

Fig. 6 presents plots that compare the bit-rates of z-DMT, CMT and FMT on TP1 lines of different lengths. Also shown in this figure are the results of an ideal system where a bank of ideal filters with zero transition bands and a channel with flat gain over each sub-band are assumed. Moreover, for CMT we have presented the results when a prototype filter with PR property (designed using the code given in [31]) is used and when the design procedure of Section IV is adopted. As seen, CMT, even with PR design, outperforms z-DMT and FMT for all the line lengths with a gain of 5 to $10 \%$ higher bit-rate. Moreover, CMT approaches very close to the upper bound of the bit-rate determined by the idealized system. A design based on PR property is already within $5 \%$ of the upper bound. The filter design proposed in Section IV reduces this gap to around $2 \sim 3 \%$. An 


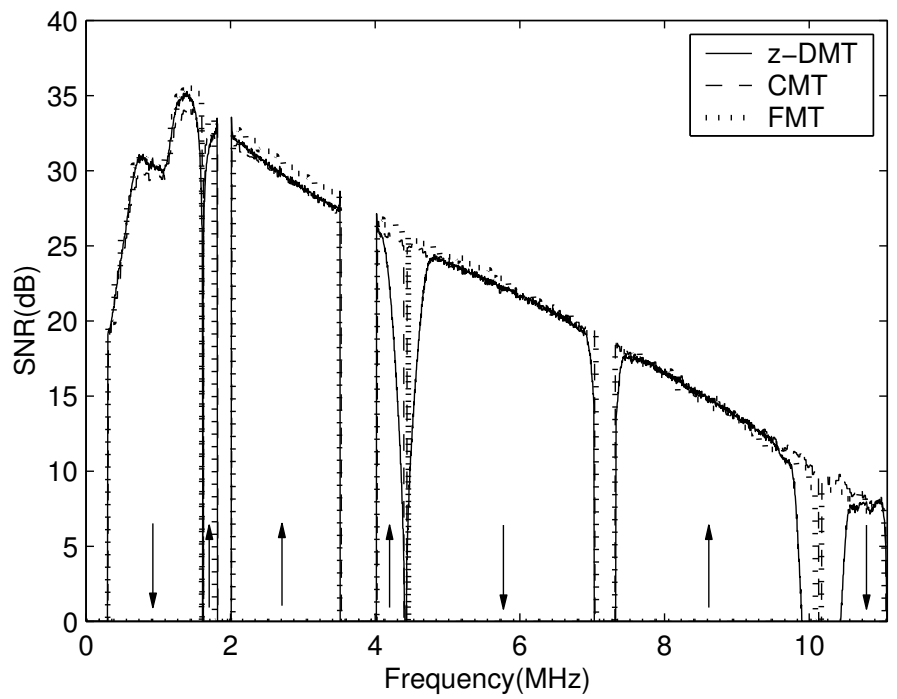

Fig. 5. SNR curves showing the impact of NEXT on z-DMT, CMT and FMT. Arrows indicate the direction of data transmission.

observation in Fig. 6 that requires some comments is that the performance of FMT is worse than that of FMT obtained in [36], especially when the length of the line is larger than $1000 \mathrm{~m}$. This is because we use a different noise model than [36]. We follow [15] and use ETSI'A' as the background noise, while $-140 \mathrm{dBm} / \mathrm{Hz}$ white Gaussian noise is used in [36].

Bit allocation for each sub-carrier is done based on the following formula[4], [33]:

$$
b_{i}=\log _{2}\left(1+\frac{\mathrm{SNR}_{i} \cdot \gamma_{\text {code }}}{\Gamma \cdot \gamma_{\text {margin }}}\right)
$$

where $\mathrm{SNR}_{i}$ is signal-to-noise ratio at the $i$ th sub-carrier, $\gamma_{\text {code }}=3 \mathrm{~dB}$ is the coding gain, $\Gamma=9.8$ $\mathrm{dB}$ is the SNR-gap between the Shannon capacity and QAM-modulation to achieve a BER of approximately $10^{-7}$, and $\gamma_{\text {margin }}=6 \mathrm{~dB}$ is the system margin. Since in CMT data symbols are PAM, we treat each pair of adjacent PAM symbols as one QAM symbol and apply (41).

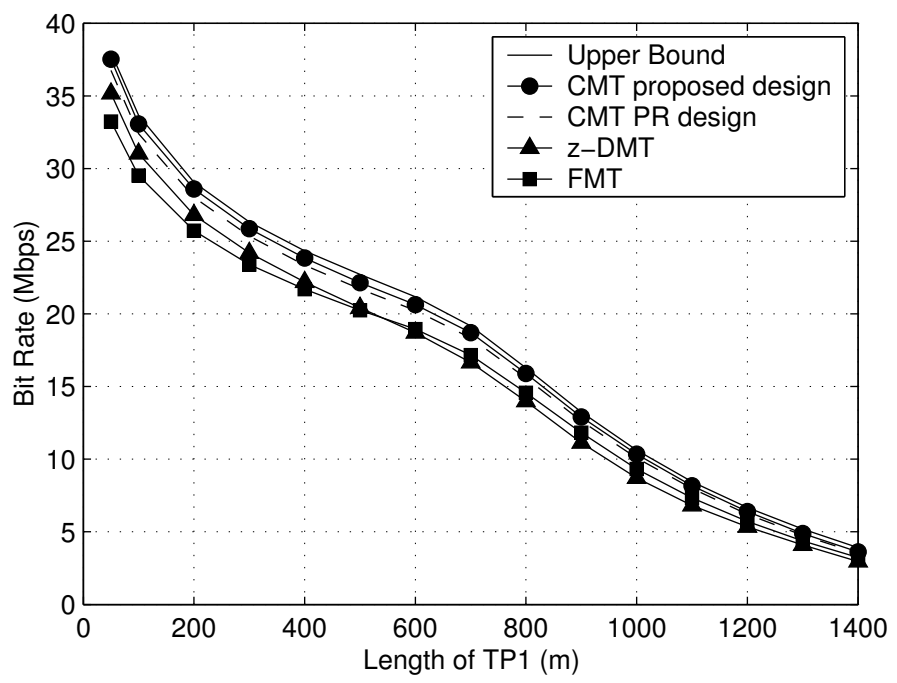

Fig. 6. Comparison of bit-rates of z-DMT, CMT and FMT on TP1 lines of different lengths. 


\section{Channels with Bridged Taps}

So far, the simulated subscriber loops were homogeneous lengths of TP1 cables. Previous reports, [25], as well as our simulation studies have shown that the group delay distortion of such lines is very minimal and mostly limited to very low and very high frequencies in the VDSL band. Nonhomogeneous subscriber lines with bridged taps, on the other hand, exhibit significant group delay distortion. Hence, a study of CMT behavior in VDSL loops with bridged taps is essential to complete our study. We present simulation results for the five test loops that are shown in Figs. 7. These are chosen from the test loops provided in [37]. Fig. 8 presents the group delays of two of these loops and also that of a $300 \mathrm{~m}$ TP1 line with no bridged tap. We note that the line without bridged tap exhibit almost no group delay distortion over most of the channel band, while as the number of bridged taps increases the group delay distortion also increases. We also note that the fast variations of the group delay at certain frequencies coincide with the points where the magnitude gain of the channel is reduced due to signal reflection from the open-ended bridged tap extensions. This phenomena is clearly seen by referring to Fig. 9 where the sub-carrier SNRs of z-DMT, CMT and FMT are shown for the loop 4 'short'. The following observations are also made by referring to Fig. 9. Even though the group delay distortion may bring some degradation to the CMT performance since it affects the flatness of each sub-channel, this degradation is not significant. It is worth noting that the sharp variations of the group delay at frequencies (about) 0.6 and $1.3 \mathrm{MHz}$, in Fig. 8, coincide with the sharp drops in SNRs of all the three systems in Fig. 9. The fact that both CMT and z-DMT behave similarly, at these points, and also recalling that DMT has no sensitivity to group delay distortion clearly indicate that the variation of group delay, in VDSL channels, has little effect in degrading the performance of CMT. On the other hand, bit rate evaluations presented in Table IV reveal that even for such extreme lines, CMT is superior to z-DMT and FMT.
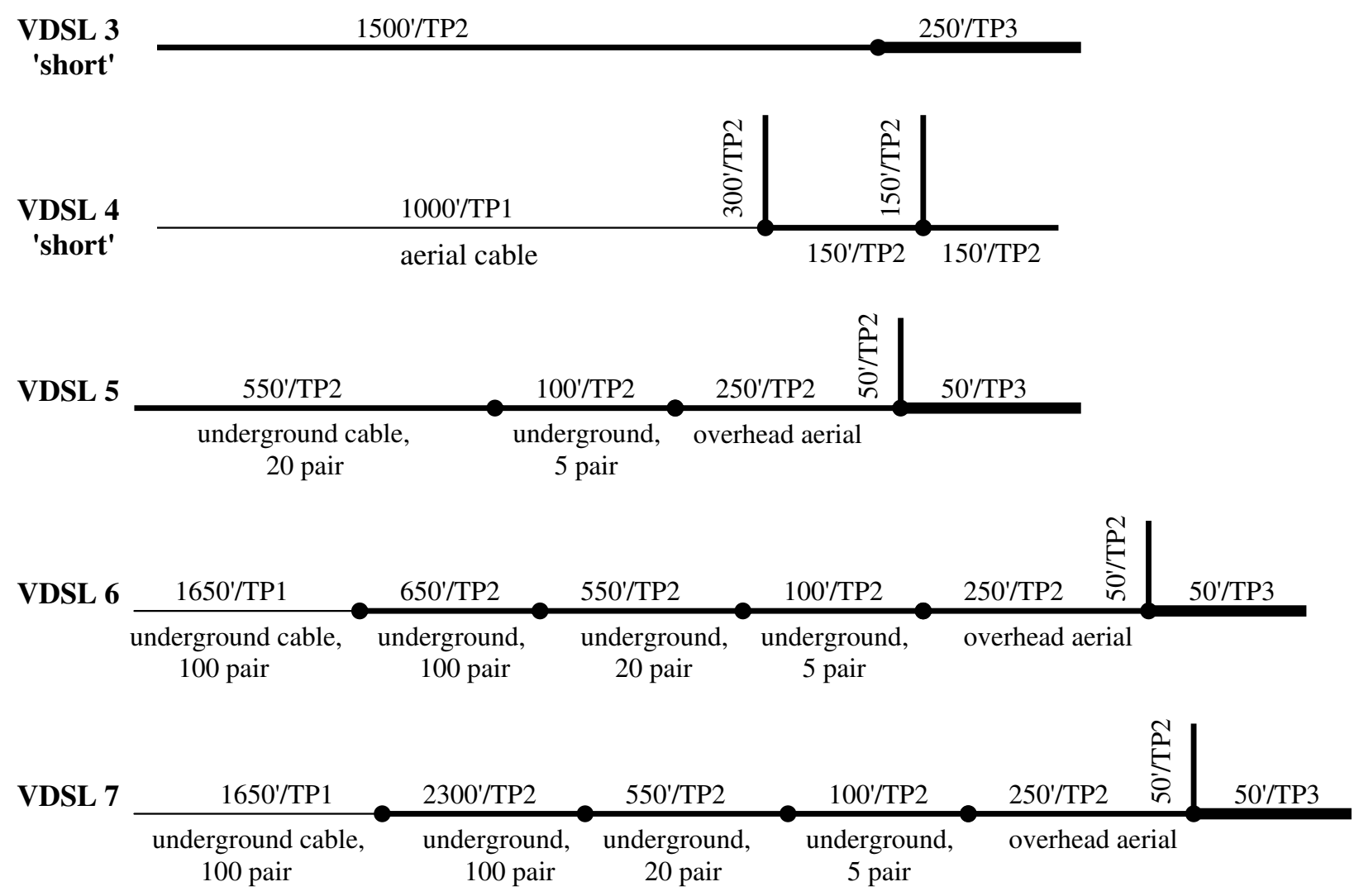

Fig. 7. Examples of test loops with bridged taps. 


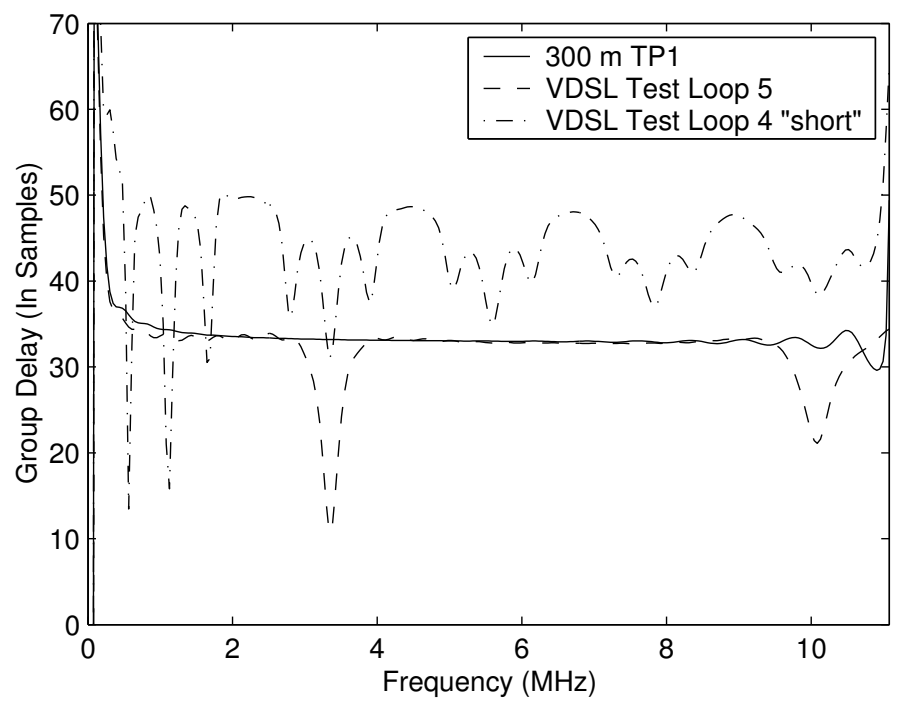

Fig. 8. Group delays of the test loops shown in Fig. 7 and a TP1 line of length $300 \mathrm{~m}$.

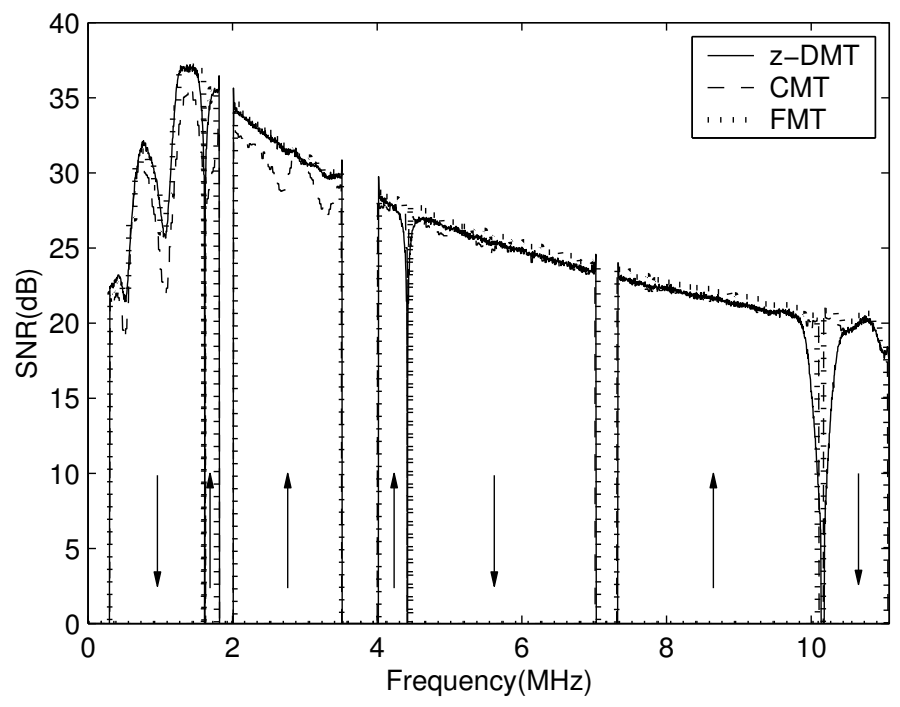

Fig. 9. SNR plots of z-DMT, CMT and FMT for the VDSL test loop 4 'short'. The plots confirm that group delay distortion in this loop has no significant impact on degrading CMT performance when compared with z-DMT. Arrows indicate the direction of data transmission.

\section{Effect of RFI Ingress Noise}

The RFI noise can badly affect the performance of the VDSL systems as it may appear at a level much higher than the VDSL signal. The RFI has to be suppressed at two stages. The first stage uses an analog RFI suppressor at the receiver input [19]. It has been reported that this technique can result in an RFI suppression of 20 to $25 \mathrm{~dB}$ [18]. However, unfortunately, this suppression is not sufficient for an acceptable performance of z-DMT system. It is thus proposed that further suppression of RFI has to be made at the demodulator output [16], [17]. Here, we consider the RFI cancellation method proposed in [16]. In this method, the center frequency of the RFI is estimated by locating the peak of the signal within the set of tones in the HAM bands. It then uses two listener tones, one on each side of the RFI, to estimate this ingress and interpolate the RFI through the transfer function of the receiver window (see [16] for details). In our simulations we follow [16] and set the listener tones to be at 8 tone spacing from the center frequency of the RFI. 
TABLE IV

Comparison of Bit-rates (MbPs) of Z-DMT, CMT ANd FMT OVER BRIDGEd Loops

\begin{tabular}{|c|c|c|c|}
\hline & z-DMT & FMT & CMT \\
\hline VDSL 3 'short' & 20.39 & 20.08 & 21.99 \\
\hline VDSL 4 'short' & 19.21 & 19.13 & 20.05 \\
\hline VDSL 5 & 24.12 & 23.67 & 25.52 \\
\hline VDSL 6 & 9.84 & 10.58 & 11.96 \\
\hline VDSL 7 & 2.92 & 3.24 & 3.60 \\
\hline
\end{tabular}

In CMT and FMT, the sharp roll-off and the high stop-band attenuation of the analysis filters allow cancellation of the RFI without resorting to any additional post demodulator RFI canceller (i.e., the second stage of the RFI canceller). However, we note that to get an acceptable performance, the first stage of RFI suppression is needed for CMT and FMT system, as well.

Figs. 10(a) and (b) present a set of results that compare the performance of z-DMT, CMT and FMT in the presence of RFI. In both cases the RFI power has been set equal to $-35 \mathrm{dBm}$ at the demodulator input. This is assumed to be the residual from a $-10 \mathrm{dBm}$ RFI (stipulated in [37]), after the first stage of suppression. The RFI is chosen to be a $4 \mathrm{kHz}$ narrowband signal. In Fig. 10(a), the center frequency of the RFI is at $1.9 \mathrm{MHz}$. This is near the center of the first HAM band. We observe that in this case the RFI canceller clears RFI almost perfectly. There is only slight degradation in SNRs near the band edges. However, the RFI canceller fails when the RFI center frequency moves to a point near one of the VDSL signal band edges. This is shown in Fig. 10(b) where the center frequency of the RFI is shifted to $1.82 \mathrm{MHz}$. The reason for the failure of the RFI canceller in this case is that one of the listener tones used to measure RFI coincides with the VDSL signal. According to [16], as well as our simulations, any attempt to shift the listener tone nearer to the center frequency of the RFI will result in a significant degradation of the tone estimates and thus equally result in failure of the RFI canceller.

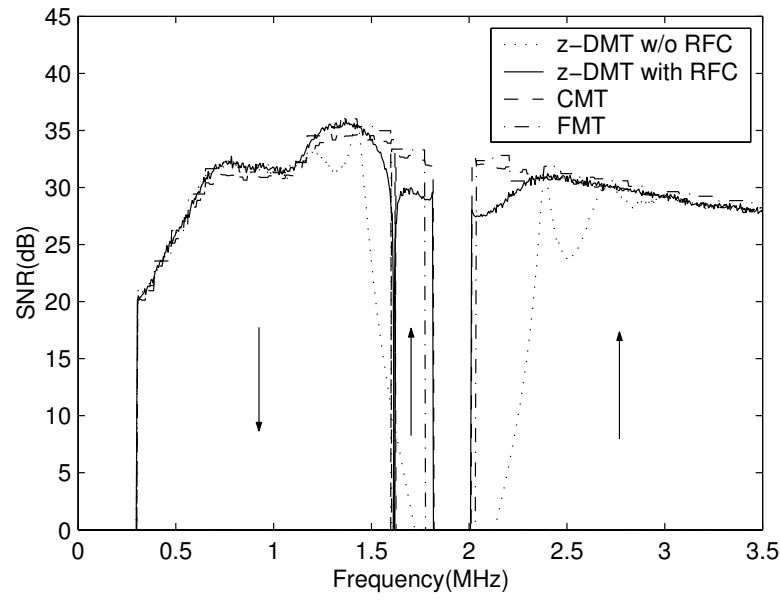

(a)

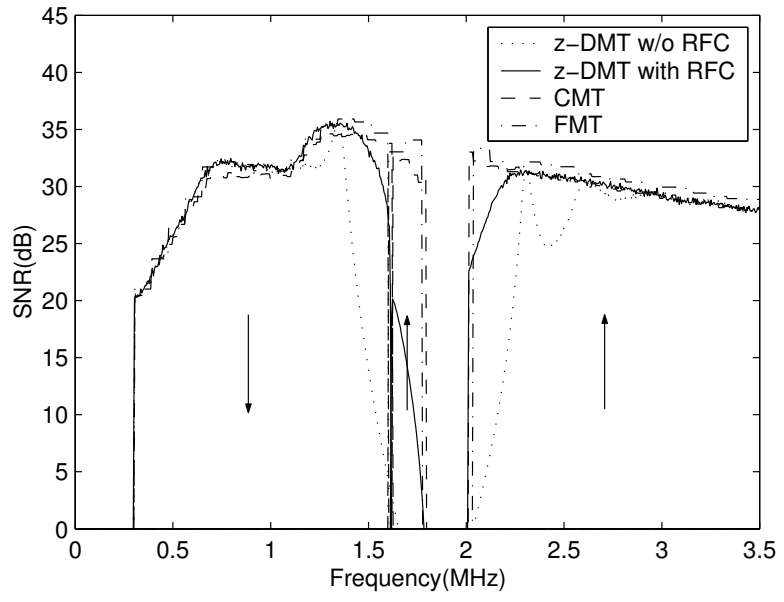

(b)

Fig. 10. RFI performance of z-DMT, CMT and FMT when an RFI with bandwidth of $4 \mathrm{kHz}$ at the level of $-35 \mathrm{dBm}$ presents at the center frequency (a) $1.9 \mathrm{MHz}$ and (b) $1.82 \mathrm{MHz}$. Arrows indicate the direction of data transmission. 


\section{Conclusions}

A thorough study of a new multi-carrier modulation in VDSL channels was presented. This modulation which uses cosine modulated filter banks was called CMT - an acronym for cosine modulated multitone. Compared to the earlier publications on the subject [40], [41], the receiver structure of CMT was modified to reduce its computational complexity. A new criterion that balances between ISI plus ICI and the stopband attenuation was proposed for designing NPR prototype filters for CMT. Numerical results showed that this criterion leads to designs that are superior to those that are designed based on the PR criterion. Moreover, CMT was compared with z-DMT and FMT, the two candidate modulation schemes for VDSL [37]. Comparisons were made with respect to computational complexity, latency, achievable bit-rates, and resistance to cross-talks and RFI. Except computational complexity, where CMT was found to be more complex than z-DMT, CMT showed superior performance with all other respects. Compared to FMT, CMT was found to be superior with respect to computational complexity and achievable bit-rate. CMT and FMT showed similar resistance to cross-talks and RFI, and had similar latency.

We note that the CMT scheme that was proposed in this paper is nothing but an amended version of DWMT, a modulated scheme which has been known for a decade [22]. However, because of its relatively high computational complexity, which was a consequence of inappropriate selection of the receiver structure, DWMT was never accepted by the industry. We hope that this revisit of the scheme and in particular the simplification of the receiver structure that is proposed in this paper can initiate new thoughts on reconsideration of this powerful signal processing tool in xDSL applications. 


\section{Appendix: Prototype Filter Design}

The MATLAB function below can be used to design a prototype filter based on the design criterion discussed in Section IV. Note that to grantee the stability of the design the stopband edge frequency $f_{\mathrm{s}}=\omega_{\mathrm{s}} / 2 \pi$ should be limited to the range $1 /(2 M)$ to $3.8 /(2 M)$. Also, the parameter $\gamma$ is initialized to 1 and progressively increase of a specified maximum as the design proceeds. We have experimentally found that this procedure always leads to good design within small number of iterations.

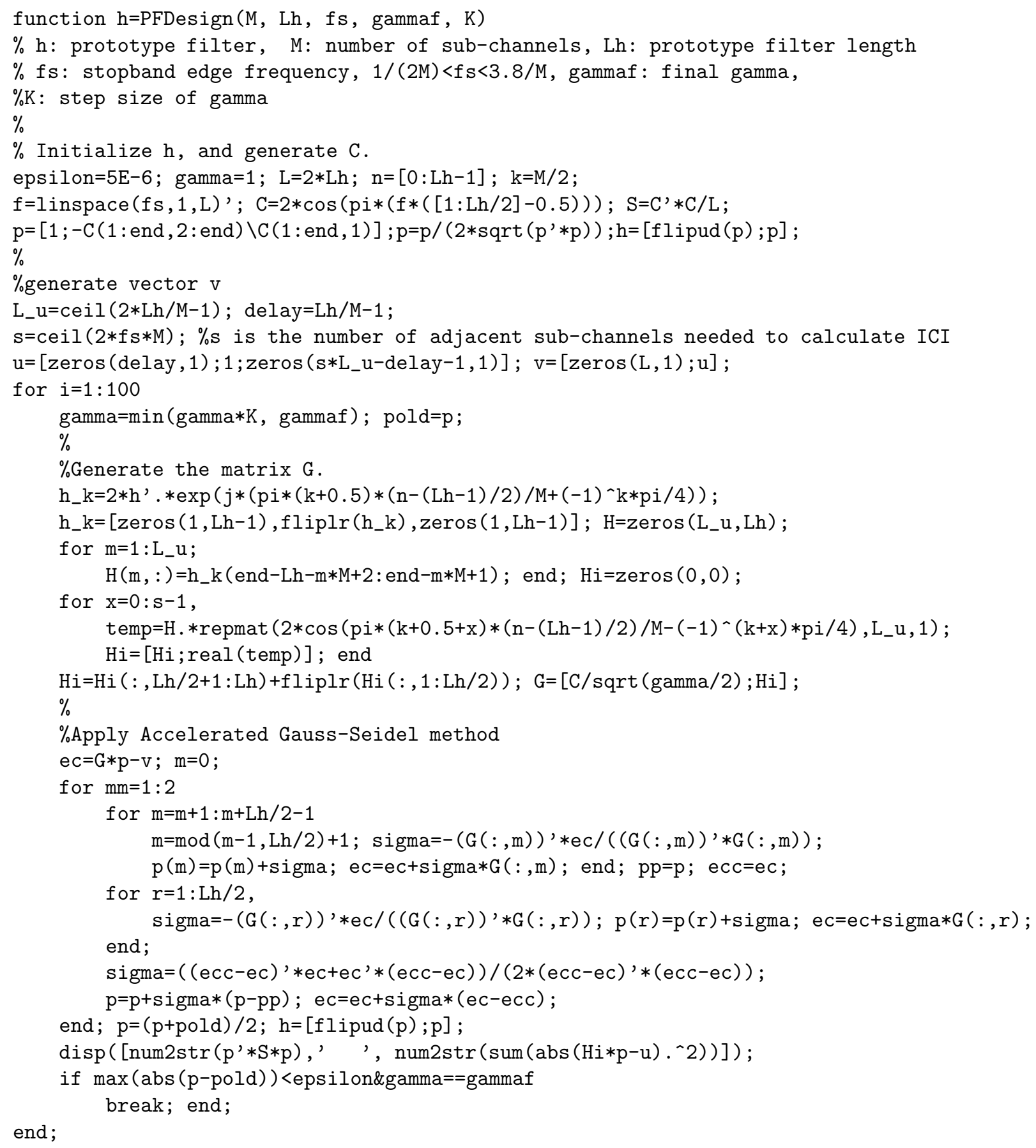




\section{REFERENCES}

[1] E. A. Lee, and D. G. Messerschmitt, Digital Communication, 2nd Ed., Kluwer, Boston, 1994.

[2] J.G. Proakis, Digital Communications, 3rd Edition, New York: McGraw Hill, 1995.

[3] Walter Y. Chen, DSL: Simulation Techniques and Standards Development for Digital Subscriber Line Systems, Macmillan Publishing, Indianapolis, Indiana, 1998.

[4] T. Starr, J.M. Cioffi, and P.J. Silverman, Understanding Digital Subscriber Line Technology, Upper Saddle River, NJ: Prentice Hall, 1999.

[5] R. Steele, Mobile Radio Communications, New York: IEEE Press, 1992.

[6] J.A.C. Bingham "Multicarrier modulation for data transmission: an idea whose time has come," IEEE Commun. Mag., vol. 28, no. 5, pp. 5-14, May 1990.

[7] xDSL Forum, http://www.dslforum.org.

[8] ETSI, http://www.etsi.org.

[9] ANSI T1E1.4 Working Group, http://www.t1.org.

[10] "Network and Customer Installation Interfaces - Asymmetric Digital Subscriber Line (ADSL) Metallic Interface," T1.413-1998, American National Standards Institute, NY, 1998.

[11] "Radio broadcasting systems; digital audio broadcasting (DAB) to mobile, portable and fixed receivers," ETS 300 401, European Telecommunications Standards Institute, 2nd ed., May 1997.

[12] "Digital Video Broadcasting (DVB); Framing structure, channel coding and modulation for digital terrestrial television," ETS 300 744, European Telecommunications Standards Institute, March 1997.

[13] F. Sjoberg, M. Isaksson, R. Nilsson, P. Odling, S.K. Wilson, and P.O. Borjesson, "Zipper: A duplex method for VDSL based on DMT," IEEE Trans. Commun., vol. 47, No. 8, pp. 1245-1252, Aug. 1999.

[14] D. G. Mestdagh, M. R. Isaksson, and P. Odling, "Zipper VDSL: a solution for robust duplex communication over telephone lines," IEEE Commun. Mag., vol. 38, no. 5, pp. 90-96, May 2000.

[15] F. Sjoberg, R. Nilsson, M. Isaksson, P. Odling, and P.O. Borjesson, "Asynchronous Zipper," in Proc. ICC'99, vol. 1, pp. 231-235, 1999.

[16] R. Nilsson, Digital Communication in Wireline and Wireless Environments, PhD Thesis, LuleåUniversity of Technology, March 1999, available at http://www.sm.luth.se/csee/sp/publications.html\#Theses.

[17] B-J Jeong and K-H Yoo, "Digital RFI canceller for DMT-based VDSL," Electronics Letters, vol. 34, no. 17, pp. 1640-1641, Aug. 1998.

[18] J. Cioffi, M. Mallory, and J. Bingham, "Digital RF cancellation with SDMT," ANSI Contribution T1E1.4/96083, April 1996.

[19] J. Cioffi, M. Mallory, and J. Bingham, "Analog RF cancellation with SDMT," ANSI Contribution T1E1.4/96084, April 1996.

[20] M.A. Tzannes, M.C. Tzannes, and H. Resnikoff, "The DWMT: A Multicarrier Transceiver for ADSL using M-band Wavelet Transforms," ANSI Contribution T1E1.4/93-067, March 1993.

[21] M.A. Tzannes, M.C. Tzannes, J. Proakis, and P.N. Heller, "DMT systems, DWMT systems and digital filter banks," in Proc. SUPERCOMM/ICC'94, vol.1, pp. 311-315, 1994.

[22] S.D. Sandberg and M.A. Tzannes, "Overlapped Discrete Multitone Modulation for High Speed Copper Wire Communications," IEEE Journal on Selected Areas in Commun., vol. 13, no. 9, pp. 1571-1585, Dec. 1995.

[23] M. Hawryluck, A. Yongacoglu, and M. Kavehrad, "Efficient Equalization of Discrete Wavelet Multitone over Twisted Pair," in Proc. Int. Zurich Seminar on Broadband Commun.'98, pp. 185-191, Zurich, 1998.

[24] N. Neurohr and M. Schilpp, "Comparison of transmultiplexers for multicarrier modulation," in Proc, ICSP'98, vol. 1, pp. 35-38, 1998.

[25] A. Viholainen, J. Alhava, J. Helenius, J. Rinne, and M. Renfors, "Equalization in filter bank based multicarrier systems," in Proc. ICECS'99, vol. 3, pp. 1467-1470, 1999.

[26] S. Govardhanagiri, T. Karp, P. Heller, and T. Nguyen, "Performance analysis of multicarrier modulation systems using cosine modulated filter banks," in Proc. ICASSP'99, vol. 3, pp. 1405-1408, 1999.

[27] B. Farhang-Boroujeny, and W.H. Chin, "Time domain equaliser design for DWMT multicarrier transceivers," Electronics Letters, vol. 36, no. 18, pp. 1590-1592, 31 Aug. 2000.

[28] P.P. Vaidyanathan, Multirate Systems and Filter Banks, Englewood Cliffs, New Jersey, Prentice Hall, 1993.

[29] H.S. Malvar, Signal Processing with Lapped Transforms, Norwood, MA: Artech House, 1992.

[30] B. Farhang-Boroujeny, Adaptive Filters: Theory and Applications, John Wiley \& Sons, Chichester, England, 1998.

[31] M. Rossi, J.Y. Zhang, and W. Steenaart, "Iterative least squares design of perfect reconstruction QMF banks," in Proc. Canadian Conference on Electrical and Computer Engineering, vol. 2, pp. 762-765, 1996.

[32] B. Borna and T.N. Davidson, "Efficient filter bank design for filtered multitone modulation", in Proc. IEEE International Conference on Communications, vol. 1, pp. 38-42, 2004.

[33] J. M. Cioffi, "A Multicarrier Primer," ANSI Contribution T1E1.4/91-157, Nov. 1991.

[34] G. Cherubini, E. Eleftheriou, S. Olcer, "Filtered multitone modulation for VDSL," in Proc. IEEE Globecom'99, vol. 2, pp. 1139-1144, 1999. 
[35] G. Cherubini, E. Eleftheriou, S. Olcer, J.M. Cioffi, "Filter bank modulation techniques for very high speed digital subscriber lines," IEEE Commun. Mag., vol. 38, no. 5, pp. 98-104, May 2000.

[36] G. Cherubini, E. Eleftheriou, S. Olcer, "Filtered multitone modulation for very high-speed digital subscriber lines," IEEE Journal on Selected Areas in Commun., vol. 20, no. 5, pp. 1016-1028, June 2002.

[37] Committee T1, Working Group T1E1.4, "VDSL Metallic Interface: Part1 - Functional rquirements and common specifications," Draft Standard, T1E1.4/2000-009R3, Feb. 2001; and "VDSL Metallic Interface: Part 3 - Technical specification of multi-carrier modulation transceiver," Draft Standard, T1E1.4/2000-013R1, May 2000.

[38] G. W. Wornell, "Emerging applications of multirate signal processing and wavelets in digital communications," Proceedings of the IEEE, vol. 84, no. 4, pp. 586-603, April 1996.

[39] B. Farhang-Boroujeny and L. Lin, "Analysis of post-combiner equalizers in cosine modulated filter bank based transmultiplexer systems," IEEE Trans. Signal Proc., vol. 51, no. 12, pp. 3249-3262, Dec. 2003.

[40] B. Farhang-Boroujeny, "Multicarrier modulation with blind detection capability using cosine modulated filter banks," IEEE Trans. Commun., vol. 51 ,no. 12, pp. 2057-2070, Dec. 2003.

[41] B. Farhang-Boroujeny, "Discrete multitone modulation with blind detection capability," in Proc. of Vehicular Technology Conference, Vancouver, Canada, Sept. 2002.

[42] J. Alhava and M. Renfors, "Adaptive sine-modulated/cosine-modulated filter bank equalizer for transmultiplexers," in Proc. Eur. Conf. on Circuit Theory and Design, Espoo, Finland, Aug. 2001.

[43] A.Viholainen, J. Alhava and M. Renfors, "Implementation of parallel cosine and sine modulated filter banks for equalized transmultiplexer systems", in Proc. ICASSP'01, vol. 6, pp. 3625-3628, May, 2001.

[44] J. Alhava and M. Renfors, "Exponentially-modulated filter bank-based transmultiplexer", in Proc. ISCAS'03, vol. 4, pp. IV-233-IV-236, May, 2003.

[45] A. Scaglione, G.B. Giannakis, and S. Barbarossa, "Redundant filterbank precoders and equalizers, Part I: unification and optimal designs," IEEE Trans. Signal Proc., vol. 47, no. 7, pp. 1988-2006, July 1999.

[46] Y-P. Lin and S-M Phoong, "ISI-free FIR filterbank transceivers for frequency-selective channels," IEEE Trans. Signal Proc., vol. 49, no. 11, pp. 2648-2658, Nov. 2001.

[47] A. Bjorck, Numerical Methods for Least Squares Problems, SIAM, Philadelphia, 1996.

[48] C. Brezinski and L. Wuytack, Projection methods for Systems of Equations, Elsevier Science B.V, Amsterdam, 1997.

[49] T. M. Ng, B. Farhang-Boroujeny and H. K. Garg, "An accelerated Gauss-Seidel method for inverse modeling", Signal Processing, vol. 83, pp. 517-529, March, 2003.

[50] "Transmission and Multiplexing (TM); Access transmission systems on metallic cables: Very high speed Digital Subscriber Line (VDSL); Part1: Functional requirements," Technical Specification TS 101 270-1 V1.1.1 (199804), ETSI, 1998.

[51] L. Lin, Multicarrier Communications Based on Cosime Modulated Filter Banks. PhD Thesis, Univesity of Utah, To be submitted. 